\title{
Corneal Cross-Linking: The Evolution of Treatment for Corneal Diseases
}

\author{
Duoduo Wu ${ }^{1}$, Dawn Ka-Ann Lim ${ }^{1,2}$, Blanche Xiao Hong Lim ${ }^{1,2}$, Nathan Wong ${ }^{3}$, \\ Farhad Hafezi ${ }^{4,5,6,7,8+}$, Ray Manotosh ${ }^{1,2}$ and Chris Hong Long Lim ${ }^{1,2,9,10 † *}$
}

${ }^{1}$ Yong Loo Lin School of Medicine, National University of Singapore, Singapore, Singapore, ${ }^{2}$ Department of Ophthalmology, National University Health System, Singapore, Singapore, ${ }^{3}$ Royal Victorian Eye Hospital, Melbourne, VIC, Australia, ${ }^{4}$ Ocular Cell Biology Group, Center for Applied Biotechnology and Molecular Medicine, University of Zurich, Zurich, Switzerland, ${ }^{5} E L Z A$ Institute, Dietikon, Switzerland, ${ }^{6}$ Faculty of Medicine, University of Geneva, Geneva, Switzerland, ${ }^{7}$ Ophthalmology, USC Roski Eye Institute, Los Angeles, CA, United States, ${ }^{8}$ Ophthalmology, Wenzhou Medical University, Wenzhou, China, ${ }^{9}$ Singapore Eye Research Institute, Singapore, Singapore, ${ }^{10}$ School of Optometry and Vision Science, University of New South Wales, Sydney, NSW, Australia

Corneal cross-linking (CXL) using riboflavin and ultraviolet A (UVA) light has become a useful treatment option for not only corneal ectasias, such as keratoconus, but also a number of other corneal diseases. Riboflavin is a photoactivated chromophore that plays an integral role in facilitating collagen crosslinking. Modifications to its formulation and administration have been proposed to overcome shortcomings of the original epitheliumoff Dresden CXL protocol and increase its applicability across various clinical scenarios. Hypoosmolar riboflavin formulations have been used to artificially thicken thin corneas prior to cross-linking to mitigate safety concerns regarding the corneal endothelium, whereas hyperosmolar formulations have been used to reduce corneal oedema when treating bullous keratopathy. Transepithelial protocols incorporate supplementary topical medications such as tetracaine, benzalkonium chloride, ethylenediaminetetraacetic acid and trometamol to disrupt the corneal epithelium and improve corneal penetration of riboflavin. Further assistive techniques include use of iontophoresis and other wearable adjuncts to facilitate epithelium-on riboflavin administration. Recent advances include, Photoactivated Chromophore for Keratitis-Corneal Cross-linking (PACK-CXL) for treatment of infectious keratitis, customised protocols (CurV) utilising riboflavin coupled with customised UVA shapes to induce targeted stiffening have further induced interest in the field. This review aims to examine the latest advances in riboflavin and UVA administration, and their efficacy and safety in treating a range of corneal diseases. With such diverse riboflavin delivery options, CXL is well primed to complement the armamentarium of therapeutic options available for the treatment of a variety of corneal diseases.

Keywords: corneal cross-linking, customised CXL, infectious keratitis, keratoconus, myopia

\section{INTRODUCTION}

Corneal ectasias like keratoconus, pellucid marginal degeneration, and post-refractory or posttraumatic corneal ectasia are characterised by progressive corneal steepening, thinning and refractive changes. These disorders can severely impede an individual's sight and quality of life and are potentially disabling. 
Conventional non-surgical management of corneal ectasias consists of conservative options such as spectacles and contact lenses. Hard contact lenses, such as scleral or hybrid contact lenses, may be prescribed. Classic surgical options include penetrating keratoplasty, deep anterior lamellar keratoplasty or intracorneal ring segments. More recently, techniques such as the use of isolated Bowman layer transplantation and corneal allogenic intrastromal ring segment (CAIRS) implantation have been reported as effective techniques in the management of patients with keratoconus (Jacob et al., 2018; van Dijk et al., 2018; Zygoura et al., 2018). Although such approaches can be effective, they can be costly, and dependent upon skilled surgeons for good outcomes. Furthermore, corneal grafting requires patients to take long-term immunosuppressant drugs, and the procedure may place patients at an increased life-time risk of developing globe rupture due to its weakened architecture (Ross et al., 2009).

Corneal cross-linking (CXL) is a Food and Drug Administration-approved, minimally invasive intervention that utilises ultraviolet A (UVA) and riboflavin (vitamin $\mathrm{B}_{2}$ ) to slow or even halt the progression of corneal ectasias. However, CXL is evolving. With our growing understanding of this procedure, modifications have been made not only to riboflavin's formulation, but also to its delivery method and UV-A irradiation regime. Multiple assistive devices and adjunct procedures have also been designed to enhance the pharmacological effectiveness of riboflavin and UVA in inducing cross-links. This review aims to showcase the strides made to optimise and adapt CXL protocols according to the pharmacokinetic properties of riboflavin and UVA to 1) improve upon their efficacy in treating corneal diseases, 2) expand their utility to function as anti-infective therapy in the treatment of infective keratitis and, 3) reduce toxicological profile in thin corneas.

\section{The Human Cornea and Its Effect on Ultraviolet Irradiation and Oxygen Consumption}

The human cornea is an optically important structure. Its transparency enables passage of light into the retina while functioning primarily as a refractory surface, contributing approximately $70 \%$ of the eye's total refractive power. Anatomically, the cornea is made up of six distinct layers - the epithelium, Bowman's membrane, stroma, Dua's layer, Descemet's membrane, and the endothelium. The epithelial cells contain tight junction complexes to prevent ingress of paracellular fluid. Together with Bowman's layer, these structures absorb UV radiation. The stroma contains a mixture of type I and V collagen fibrils oriented in a regular fashion, accounting for $90 \%$ of the entire cornea's thickness. These interwoven fibrils are uniformly spaced by proteoglycans, forming stacked lamellae (Abahussin et al., 2009). Biomechanically, this arrangement confers viscoelasticity, protecting the cornea against deformation from environmental and intraocular pressures.

Besides its innate biomechanical structure, the cornea also contains an active fluid transport system to maintain its structural integrity. To support this metabolic system, the cornea requires a constant supply of oxygen. As an avascular structure (except for a limited peripheral zone), the cornea respires primarily across its anterior and posterior surfaces. Additionally, various components of the cornea consume varying amounts of oxygen. The epithelium utilises $40 \%$ of the total oxygen consumption of the cornea, while the stroma and endothelium consume $21 \%$ and $39 \%$ respectively. However, the per unit area of oxygen consumption of the epithelium is 10 times that of the stroma, and approximately 0.2 times that of the endothelium (Freeman, 1972).

\section{MECHANISMS OF ACTION}

The 2015 Global Consensus on Keratoconus and Ectatic Diseases highlighted riboflavin and UVA's integral role in the treatment of corneal ectasias through strengthening of the corneal stroma (Gomes et al., 2015). At the molecular level, cross-links between corneal collagen monomers can be formed through enzymatic, glycosylation or oxidative pathways. Enzymatic cross-linking reactions from lysyl oxidase occur as part of the natural ageing process while glycosylation commonly occurs in patients with diabetes mellitus as part of the Maillard reaction (Dawczynski et al., 2002). These processes may explain reduced rates of keratoconus progression among people with diabetes and those older than 40-years-old. In corneal cross-linking, UVA and riboflavin promote cross-linking through both the oxidative and glycosylation pathways (Figure 1).

\section{Oxidative Pathway}

A Type 1 (non-oxygen mediated) oxidative process occurs when riboflavin absorbs UV light, converting into an excited singlet riboflavin molecule that transforms into a triplet state (Raiskup and Spoerl, 2013). This reactive intermediate becomes a reservoir for hydride and electron transfer, creating covalent bonds between collagen fibrils and stromal substrates. A Type 2 (oxygen mediated) oxidative reaction occurs when UVA generates reactive oxygen species, which promotes formation of intra- and intermolecular covalent bonds to convert collagen monomers into cross-linked polymers. An understanding of these processes has been crucial in unveiling factors influencing the efficacy of CXL (Seiler et al., 2020).

\section{Glycosylation Pathway}

Apart from oxidative reactions, riboflavin and UVA also promote glycosylative cross-linking within proteoglycans and collagen fibrils via advanced glycation end product-mediated mechanisms (Brummer et al., 2011). Additionally, riboflavin further functions as an optical buffer for UVA. It absorbs UVA, thereby reducing the risk of damage to posteriorly located structures such as the corneal endothelium, crystalline lens and retina (Wollensak et al., 2010).

\section{DRESDEN PROTOCOL}

Epithelium-off corneal cross-linking, or termed "Dresden protocol," is the first widely recognised CXL protocol introduced by Dr. Theo Seiler and his team. This involves riboflavin $0.1 \%$ in $20 \%$ dextran and a UVA light source $(365-370 \mathrm{~nm})$ (Spoerl et al., 1998). Experimental animal 


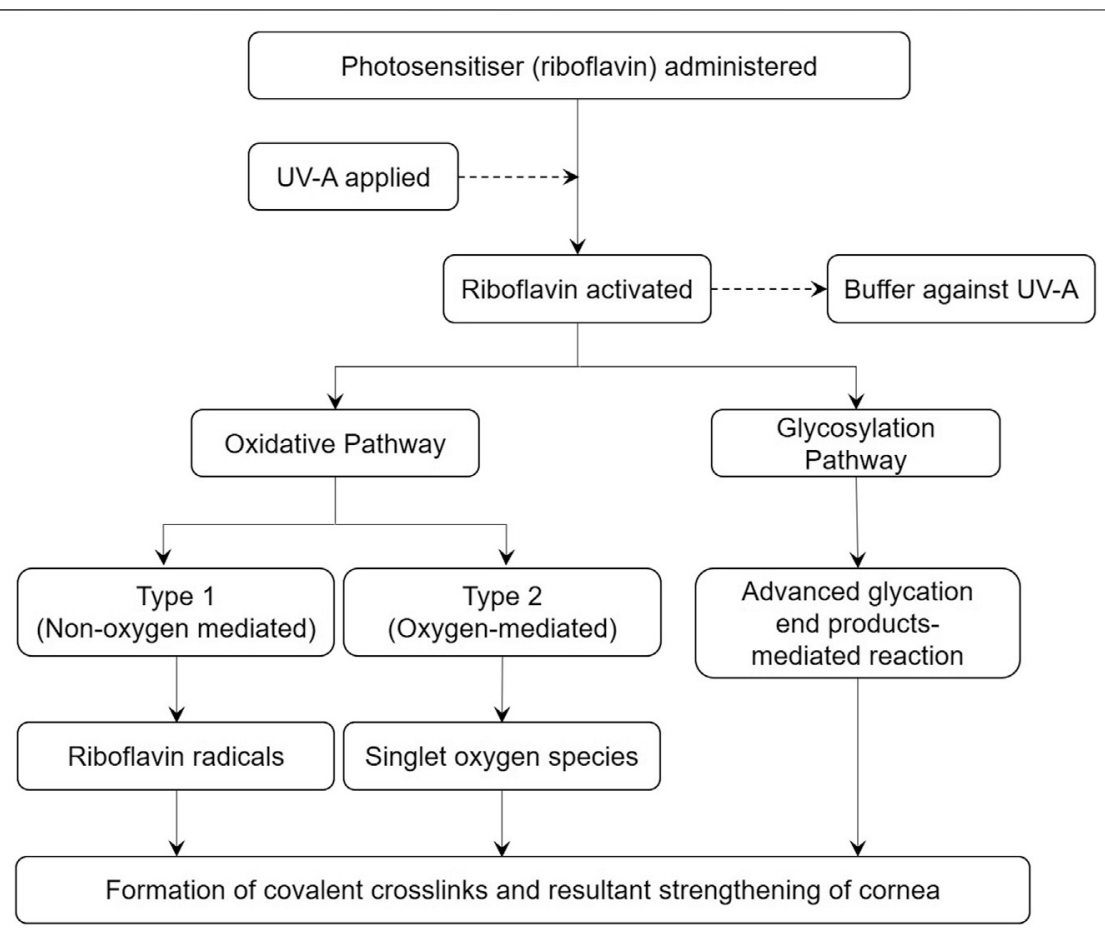

FIGURE 1 | Proposed mechanisms of action of riboflavin.

studies have demonstrated increased cornea rigidity of between $42 \%$ and $70 \%$ in corneas treated with riboflavin and UVA (Seifert et al., 2014; Matteoli et al., 2016). Topical anaesthesia is administered to the ocular surface before debridement of the central 7-9 mm of corneal epithelium. Riboflavin is subsequently administered topically every 2 minutes for 30 minutes. Following which, irradiance of the central cornea of $3 \mathrm{~mW} / \mathrm{cm}^{2}$, for a treatment duration of 30 minutes and surface dose of $5.4 \mathrm{~J} /$ $\mathrm{cm}^{2}$ was performed. Riboflavin is applied at 5 minute intervals throughout the treatment duration. Post-treatment, antibiotic eye drops are administered, and a bandage contact lens is placed over the cornea. Patients are instructed to administer topical antibiotics and steroids following this procedure.

Efficacy of the standard protocol in halting progression of keratectasia has been validated by multiple short-term and longterm studies. Current evidence shows that progression of keratoconus can be halted, with sustained improvements in clinical outcomes demonstrated for up to 10 years of followup (Raiskup and Spoerl, 2013; Wittig-Silva et al., 2014; Seyedian et al., 2015; Taşçı et al., 2020).

\section{MAXIMISING PHARMACOKINETIC PROPERTIES OF RIBOFLAVIN/ ULTRAVIOLET-A IN THE TREATMENT OF KERATOCONUS}

To maximise treatment efficacy, safety and comfort, several modifications have been made according to the pharmacokinetic properties of riboflavin and UVA: 1) UVA irradiation, 2) riboflavin's route of administration, 3) oxygen supplementation and 4) introduction of assistive modalities to improve visual acuity (Table 1; Figure 2).

\section{UVA Irradiation}

UVA interacts with riboflavin to activate oxidative and glycosylation pathways that lead to the formation of collagen cross-links. A dosage (fluence) of $5.4 \mathrm{~J} / \mathrm{cm}^{2}$ is required to achieve the desired cornea stiffening. The original Dresden protocol utilises UVA irradiation at $3 \mathrm{~mW} / \mathrm{cm}^{2}$ for a treatment duration of 30 minutes. In a bid to improve patient comfort, "accelerated" protocols have been proposed.

\section{Accelerated Protocols}

"Accelerated" protocols have been developed to deliver higher UVA irradiance at a shorter duration to reduce patient discomfort. Several variations in UVA exposure have been described to achieve a cumulative dose of $5.4 \mathrm{~J} / \mathrm{cm}^{2}: 30 \mathrm{~mW} /$ $\mathrm{cm}^{2}$ for 3 minutes, $18 \mathrm{~mW} / \mathrm{cm}^{2}$ for 5 minutes or $9 \mathrm{~mW} / \mathrm{cm}^{2}$ for 10 minutes. This is based on Bunsen-Roscoe's law of photochemical reciprocity, which describes a linearly proportional photochemical effect to the total UV energy delivered, regardless of duration of administration (Schindl et al., 2001). A prospective non-randomised interventional study of 156 eyes with early progressive keratoconus demonstrated that accelerated epithelium-off CXL with UVA irradiation of $9 \mathrm{~mW} / \mathrm{cm}^{2}$ for 10 minutes was associated with favorable outcomes (Mazzotta et al., 2021). The 5 years results demonstrated sustained improvements in uncorrected distance 
TABLE 1 | Summary of existing corneal cross-linking protocols.

\begin{tabular}{|c|c|c|c|c|}
\hline Protocol & Riboflavin delivery & Ultraviolet-A & $\begin{array}{l}\text { Oxygen } \\
\text { delivery }\end{array}$ & Efficacy \\
\hline Dresden protocol & Epithelium-off & $3 \mathrm{~mW} / \mathrm{cm}^{2} ; 30 \mathrm{~min}$ & Room air & $\begin{array}{l}\text { Sustained clinical outcomes up to } 10 \\
\text { years postoperatively. (Raiskup and } \\
\text { Spoerl, 2013; Wittig-Silva et al., 2014; } \\
\text { Seyedian et al., 2015; Tașçı et al., 2020 }\end{array}$ \\
\hline
\end{tabular}

Ultraviolet-A irradiation

Accelerated protocol

Epithelium-off

Ultraviolet-A-Emitting device

Epithelium-on $3 \mathrm{~h} /$ day for 6 months

Riboflavin delivery

Transepithelial protocol

Transepithelial protocol with:

(1) chemical enhancers

(2) Iontophoresis

(3) Femto-second laser

Transepithelial protocol with

phonophoresis

Oral riboflavin

Improving oxygen diffusion

Pulsed Ultraviolet-A
Epithelium-on

Epithelium-on

Epithelium-on;

(1) Loosening of epithelial tight junctions

(2) electric field

(3) Femtosecond laser assisted

Epithelium-on

Exposure to $15 \mathrm{~min}$ of direct sunlight Room air everyday

$30 \mathrm{~mW} / \mathrm{cm}^{2}$ for 4 min with a $1.5 \mathrm{~s}$ on/ Room Air off cycle/Nariable

$3 \mathrm{~mW} / \mathrm{cm}^{2} ; 30 \mathrm{~min} /$ variable

$3 \mathrm{~mW} / \mathrm{cm}^{2} ; 30 \mathrm{~min} /$ variable

Room air

Room air

Epithelium-off/Nariable
Variable; $30 \mathrm{~mW} / \mathrm{cm}^{2}$ for $3 \mathrm{~min}$,

Room air $18 \mathrm{~mW} / \mathrm{cm}^{2}$ for $5 \mathrm{~min}$ or $9 \mathrm{~mW} / \mathrm{cm}^{2}$ for 10 minute

$0.31 \mathrm{~mW} / \mathrm{cm}^{2}$ for $180 \mathrm{~min}$ daily for 6 Room air months required. (Wen et al., 2018)

An experimental procedure demonstrated a statistically significant improvement in riboflavin penetration among ultrasound treated rabbit corneas $(p<0.001)$ (Lamy et al., 2013).

However, hyperthermia is a potential safety concern of this technique.

Series of three cases of keratoconus treated in this manner described no adverse effects and corneal flattening was reported within 6 months of treatment (Jarstad et al., 2019). A small prospective study is underway.

Limited data is available regarding doseresponse relationships of systemically absorbed riboflavin and its ocular bioavailability.
Stromal demarcation line was significantly deeper in the pulsed UVA group ( $213 \pm$ $47.38 \mu \mathrm{m})$ compared to the continuous UVA group (149.32 $\pm 36.03 \mu \mathrm{m})$ (Moramarco et al., 2015).

At 6 and 12 months, there was modest corneal flattening with keratometric stabilisation in $98.3 \%$ of eyes. No changes in central keratometry were noted. Moreover, mean corrected distance visual acuity, manifest refraction and endothelial cell density did not change (Gore et al., 2021)

(Continued on following page) 
TABLE 1 | (Continued) Summary of existing corneal cross-linking protocols.

\begin{tabular}{|c|c|c|c|c|}
\hline Protocol & Riboflavin delivery & Ultraviolet-A & $\begin{array}{l}\text { Oxygen } \\
\text { delivery }\end{array}$ & Efficacy \\
\hline $\begin{array}{l}\text { Enhanced-fluence pulsed- } \\
\text { light iontophoresis cross- } \\
\text { linking }\end{array}$ & Epithelium-on with iontophoresis & $\begin{array}{l}18 \mathrm{~mW} / \mathrm{cm}^{2} \text { of pulsed-light on-off } \\
\text { exposure }\end{array}$ & Room air & $\begin{array}{l}\text { At } 3 \text { years, the average uncorrected } \\
\text { distance visual acuity improved and } \\
\text { average maximum keratometry readings } \\
\text { decreased. Additionally, anterior segment } \\
\text { optical coherence tomography showed } \\
\text { that the demarcation lines were situated } \\
\text { at an average depth of } 285.8 \pm 20.2 \mu \mathrm{m} \text { in } \\
\text { more than } 80 \% \text { of patients at } 1 \text { month } \\
\text { postoperatively, a value that is close to } \\
\text { that of one created by standard } \\
\text { epithelium-off cross-linking (Mazzotta et } \\
\text { al., 2020b). }\end{array}$ \\
\hline $\begin{array}{l}\text { Periocular oxygen } \\
\text { supplementation }\end{array}$ & Epithelium-on $0.25 \%$ riboflavin & $10 \mathrm{~J} / \mathrm{cm}^{2}$ (1 s: $1 \mathrm{~s}$, pulsed) & Hyperoxic & $\begin{array}{l}\text { Aydın et al. demonstrated that patients } \\
\text { treated with periocular oxygen } \\
\text { supplemented accelerated epithelium-on } \\
\text { protocol experienced a larger decrease in } \\
\text { maximum keratometry values ( } p \text {-value = } \\
\text { 0.019) and had a significantly deeper } \\
\text { demarcation line (320 } \pm 17 \mu \mathrm{m}) \text { when } \\
\text { compared to the control group ( } 269 \pm \\
19 \mu \mathrm{m} \text { ) (Aydın and Aslan, } 2021) \text {. } \\
\text { Additionally, post-procedural endothelial } \\
\text { cell density was comparable between } \\
\text { both groups. }\end{array}$ \\
\hline
\end{tabular}

Optimising visual acuity

Customised protocol (CurV) Epithelium-off/Epithelium-on with oxygen supplementation
Customised according to corneal topography
Room air/ Hyperoxic

One year results show stronger cornea flattening and faster healing time. (Seiler et al., 2016)

Mazzotta et al. demonstrated that highirradiance epithelium-on customised CXL with supplemental oxygen induces visual improvement and flattens steep keratometry. Additionally, demarcation lines were approximately 30\% deeper in this series than previously reported by studies which utilised epithelium-off CurV protocols. This suggests the possibility of conducting CurV without the need for deepithelisation (Mazzotta et al., 2020b). Long-term studies are warranted.

Athen's protocol

Cretan protocol

Intrastromal corneal ring segment implantation with corneal cross-linking

Anti-infective application
Topographically-guided transepithelial $6 \mathrm{~mW} / \mathrm{cm}^{2} ; 10 \mathrm{~min}$ photorefractive keratectomy (PRK) followed by corneal cross-linking

Transepithelial phototherapeutic keratectomy (tPTK) with corneal cross-linking

Intrastromal corneal ring segment implantation with corneal cross-linking
$3 \mathrm{~mW} / \mathrm{cm}^{2} ; 30 \mathrm{~min}$

$9 \mathrm{~mW} / \mathrm{cm}^{2} ; 10 \mathrm{~min}$

Room air
Room air At 3 years Athen's protocol offered superior uncorrected distance visual acuity and flatter steep and flat keratometry than the standard epitheliumoff corneal cross-linking protocol. (Kymionis et al., 2009)

Room air A 3 years prospective comparative study of 30 eyes demonstrated vision improvement and mean reduction in corneal astigmatism. In comparison, patients who underwent the standard epithelium-off protocol did not have any improvements in visual acuity or corneal astigmatism (Grentzelos et al., 2019).

A large prospective study of 542 eyes showed improvements in vision and maximum keratometry value in the CXLICRS group (Singal et al., 2020). 
TABLE 1 | (Continued) Summary of existing corneal cross-linking protocols.

\begin{tabular}{|c|c|c|c|c|}
\hline Protocol & Riboflavin delivery & Ultraviolet-A & $\begin{array}{l}\text { Oxygen } \\
\text { delivery }\end{array}$ & Efficacy \\
\hline PACK-CXL & Epithelium-off & $3 \mathrm{~mW} / \mathrm{cm}^{2} ; 30 \mathrm{~min}$ & Room air & $\begin{array}{l}\text { Offered superior efficacy and healing } \\
\text { duration in treating bacterial keratitis } \\
\text { compared to antibiotics alone. (Tawfeek } \\
\text { et al., 2020) } \\
\text { Has a higher rate of corneal and worse } \\
\text { visual acuity as compared to anti-fungals } \\
\text { when treating fungal keratitis. Longer term } \\
\text { studies are warranted. (Uddaraju et al., } \\
\text { 2015; Prajna et al., 2020) }\end{array}$ \\
\hline
\end{tabular}

Thin corneas

Hypoosmolar riboflavin

Dextran-free riboflavin solution

$3 \mathrm{~mW} / \mathrm{cm}^{2} ; 30 \mathrm{~min}$

$3 \mathrm{~mW} / \mathrm{cm}^{2} ; 30 \mathrm{~min}$

Contact-lens assisted corneal cross-linking

Iso-osmolar riboflavin $0.1 \%$

Epithelium-off

Epithelial island cross-linking technique

Epi-off-lenticule-on corneal cross-linking

Pachymetry-based accelerated cross-linking

Sub400 protocol

Other indications

LASIK Xtra and SMILE Xtra
Epithelium-off

Customised pachymetry guided epithelial debridement

Epithelium-off

Various based on the nomogram

Various based on the nomogram

UV illumination time and irradiance adjusted according to the corneal thickness to achieve a safe depth of cross-linking $70 \mu \mathrm{m}$ away from the endothelium

$3 \mathrm{~mW} / \mathrm{cm}^{2} ; 30 \mathrm{~min}$
Various
Room air

Room air

Room air

Room air
Stabilised keratectasia with no resulting endothelial cell loss (Hafezi et al., 2009; Raiskup and Spoerl, 2011)

Achieved a stromal demarcation line with mean depth of $252.9 \pm 40.8 \mu \mathrm{m}$. No significant endothelial loss secondary to UVA toxicity was identified (Jacob et al., 2014).

However, the presence of a contact lens over the epithelium creates an artificial barrier that reduces oxygen diffusion into the stroma (Kling et al., 2017; Wollensak et al., 2019).

Technique performed on 19 eyes with improvement in vision, flattest keratometry and steepest keratometry values reported 1 year postoperatively. However, there was significant endothelial cell density loss $(2550 \pm 324$ vs $2030 \pm$ 200 cells $/ \mathrm{mm}^{2}$ ) 1 year postoperatively (Cagil et al., 2017).

A recent study of this technique showed that visual acuity and endothelial cell density remained stable over a 12 months follow-up. All patients were observed to have a demarcation line by 6 months follow-up (Cagini et al., 2020).

The $\mathrm{M}$ nomogram was validated against clinical findings of 20 eyes (Mazzotta et al., 2018).

However, this protocol has a distinct limitation - it requires surgeons to have access to various riboflavin formulations, and cross-linking devices that can output UVA energy at 3, 9, 15, and even $30 \mathrm{~mW} /$ $\mathrm{cm}^{2}$, using either continuous light or pulsed light protocols. Moreover, iontophoresis may even be required in some cases to perform the treatment.

Room air Pilot study showed that $90 \%$ of 39 thin corneas ranging from 214 to $398 \mu \mathrm{m}$ achieved topographical stability at 12 months, and no eyes experienced endothelial decompensation as a result of UV irradiation toxicity. (Hafezi et al., 2020)

Room air 
TABLE 1 | (Continued) Summary of existing corneal cross-linking protocols.

Protocol

Riboflavin delivery
Ultraviolet-A

Oxygen

delivery

Variable short-term results reported. Longer termed and larger scale studies required. (Kanellopoulos and Asimellis, 2015; Konstantopoulos et al., 2019;

Kohnen et al., 2020)

Hyper-osmolar riboflavin

Preoperative $40 \%$ glucose or

$3 \mathrm{~mW} / \mathrm{cm}^{2} ; 30 \mathrm{~min}$

Room air intraoperative $70 \%$ glycerol
Reduction of central corneal thickness and visual acuity observed in patients with bullous keratopathy. (Wollensak et al. 2009; Hafezi et al., 2010)

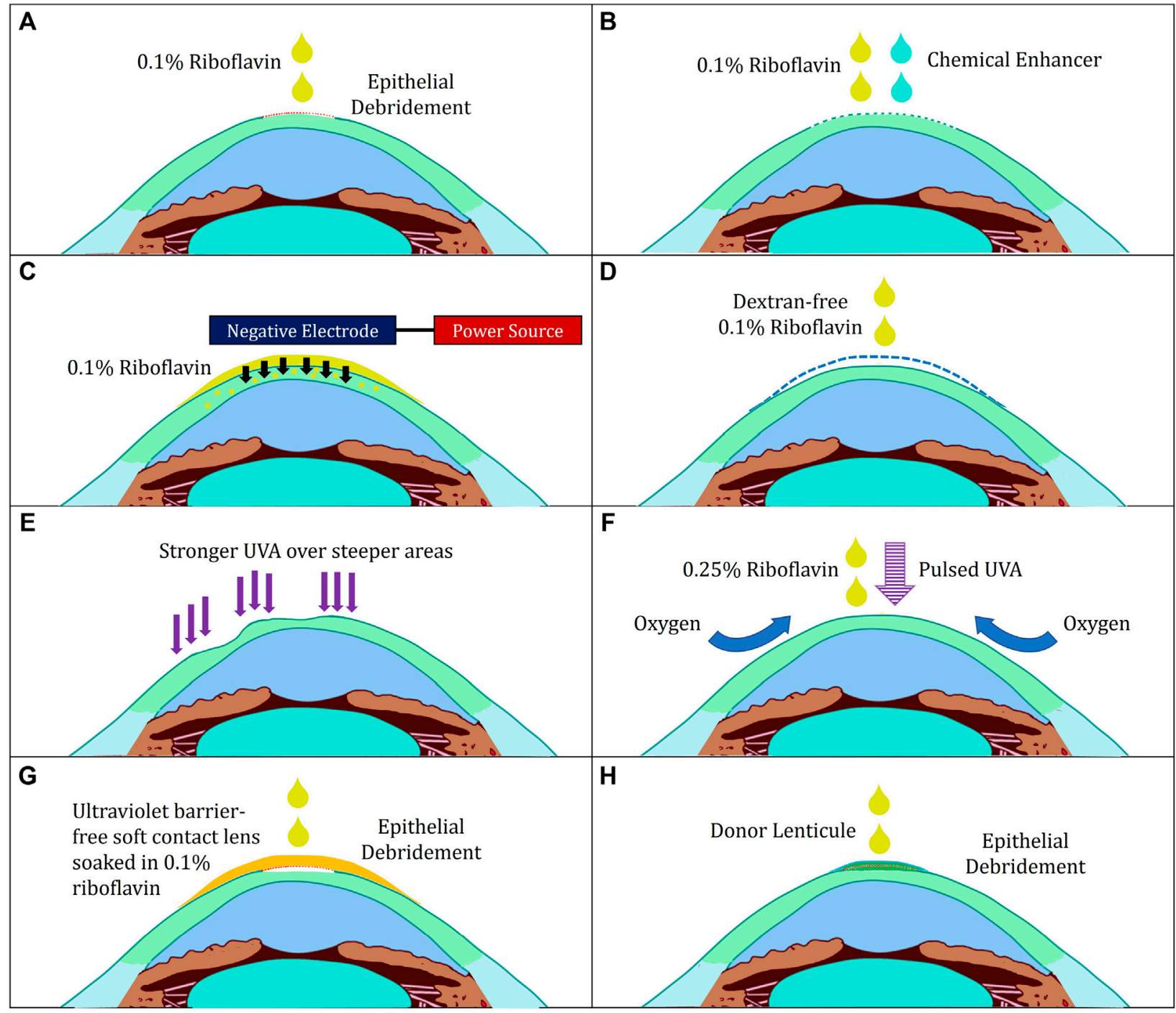

FIGURE 2 | Illustration of various modifications to corneal cross-linking. (A) Standard "epithelium-off" Dresden Protocol (B) Transepithelial Protocol with Chemical Enhancers (C) Transepithelial Protocol with lontophoresis (D) Hypoosmolar Riboflavin for Thin Corneas (E) Customised Protocol (F) Boost Epithelium-On Protocol (G) Contact-Lens Assisted Corneal Cross-Linking (H) Epi-Off-Lenticule-On Corneal Cross-linking. 
visual acuity, corrected distance visual acuity and maximum keratometry values. A mean demarcation line depth of $332.6 \pm$ $33.6 \mu \mathrm{m}$ was identified on anterior segment optical coherence tomography. Although the presence of corneal haze was noted in $11.6 \%$ of patients, this resolved in all patients following initiation of topical steroid therapy (Mazzotta et al., 2021). This study demonstrates the long-term efficacy and safety of $9 \mathrm{~mW} / \mathrm{cm}^{2}$ accelerated CXL protocols, providing evidence supporting the use of accelerated CXL protocols in the treatment of early keratoconus.

Two other prospective studies also showed keratoconus stabilisation with improvements in visual acuity after patients received accelerated CXL (Mimouni et al., 2021; Sot et al., 2021). Interestingly, Mimouni et al. reported that patients with central cones (defined as within the central $3 \mathrm{~mm}$ of the cornea) demonstrated greater improvement in best spectacle-corrected visual acuity $(0.08 \pm 0.02 \log$ MAR, $p<0.001)$ and myopia $(1.27 \mathrm{D}$ more reduction) than patients with paracentral cones (within 3-5 mm of the central cornea) (Mimouni et al., 2021). Sot et al., on the other hand, reported that $17.1 \%$ of patients in a cohort of 82 eyes treated with accelerated CXL showed signs of progression. These patients were observed to be younger, with higher maximum keratometry readings and more pronounced optical aberrations (Sot et al., 2021).

A meta-analysis comparing accelerated vs. conventional epithelium-off protocols reported stabilisation of keratometry values up to 1 year after the procedure in both groups, with no statistical difference in maximum keratometry values at 1 year of follow-up. Additionally, no statistical differences in postprocedure endothelial cell density were noted between groups. However, patients treated with the Dresden protocol exhibited better corrected visual acuity at 1 year follow-up and had a deeper demarcation line than those treated with an accelerated protocol (Kobashi and Tsubota, 2020).

A multi-centre retrospective study which included 684 eyes, examined patients undergoing both interventions and demonstrated similar outcomes (Kandel et al., 2021). Both protocols halted progression (defined as less than a dioptre increase in maximum keratometry) with similar efficacy (accelerated: $89 \%$ vs conventional: $88 \%$ ). Patients in the Dresden protocol group experienced a greater improvement in adjusted mean pinhole visual acuity ( 4.4 vs $1.6 \log \mathrm{MAR}, p$-value $=$ $0.04)$. Moreover, a higher proportion of patients in the accelerated group experienced clinically significant corneal haze (17.7 vs $10.2 \%, p$-value $=0.02$ ). While accelerated protocols provide the advantage of reducing surgical time without jeopardising short-term disease stabilisation, further studies regarding longterm outcomes are required.

Accelerated protocols commonly utilise UVA irradiance ranging between $9 \mathrm{~mW} / \mathrm{cm}^{2}$ and $30 \mathrm{~mW} / \mathrm{cm}^{2}$. However, previous clinical studies have shown that regimens of low UVA illumination with longer duration induces more crosslinks with riboflavin, implying that Bunsen-Roscoe's law does not adequately predict the efficacy of accelerated UVA regimens (Peyman et al., 2016; Choi et al., 2017; Kirgiz et al., 2019). This observation had been further supported by ex-vivo studies, demonstrating that UV fluence is non-linear for UVA illumination intensities above $9 \mathrm{~mW} / \mathrm{cm}^{2}$ (Wernli et al., 2013; Hammer et al., 2014; Lin and Cheng, 2017). Therefore, accelerated CXL protocols may have varying efficacy rates, dependent upon the UVA regimen used. Although current evidence suggests that lower irradiance produces more crosslinks, the optimal UVA regimen required to halt keratoconus progression is still not known (Seiler et al., 2020).

This observation most likely arises from reduced oxygen availability, which is crucial for the CXL reaction and its resultant biomechanical stiffening (Richoz et al., 2013). Demonstration of a significant reduction in oxygen availability during CXL at higher UVA irradiances may explain the reduced strengthening effect of accelerated protocols; where short illumination duration with high UVA intensities provides decreased time for oxygen re-diffusion to occur to produce oxygen-mediated cross-links (Seiler et al., 2020). Protocols such as the Boost Epi-On Protocol have been formulated to address these observations.

\section{Ultraviolet-A-Emitting Device}

The Dresden protocol requires patients to be stationary for an extended duration. KeraVio is a CXL treatment modality which utilises UVA emitting glasses and topical epithelium-on riboflavin administration for $3 \mathrm{~h}$ daily across 6 months. This allows patients to be ambulatory, thereby reducing patient discomfort during the procedure. Ex-vivo experiments on rabbit corneas have demonstrated that treated corneas with KeraVio were significantly stronger than controls (Kobashi et al., 2020). Clinically, KeraVio halted corneal ectasia progression without any safety concerns in 20 eyes (Kobashi et al., 2020). Further comparative studies are required to ascertain the efficacy of this technique.

\section{Riboflavin Delivery \\ Epithelium-On Protocol}

Riboflavin is a large hydrophilic molecule that does not penetrate tight junctions of the intact corneal epithelium (Gore et al., 2015). The Dresden protocol overcame this through epithelial debridement (“epithelium-off”), allowing diffusion of riboflavin molecules into the stroma. Even though epithelial debridement provides favourable riboflavin penetration, this is associated with risks such as pain, infection, persistent epithelial defects, and corneal melt (Spoerl et al., 2007; Evangelista and Hatch, 2018).

To mitigate these problems, epithelium-on protocols, where riboflavin is administered directly on an intact corneal epithelium, were devised. Although epithelium preservation increases corneal thickness and may confer protection to the underlying endothelial cells, its barrier function greatly limits the extent of riboflavin absorption, UVA penetration and oxygen availability. Consequently, epithelium-on protocols failed to demonstrate satisfactory clinical efficacy. A randomisedcontrolled trial reported evidence of keratoconus progression in $23 \%$ of eyes undergoing epithelium-on corneal crosslinking, while progression was halted in all epithelium-off eyes (Soeters et al., 2015). Meta-analyses performed by Li and Wang, and Nath et al. confirmed that epithelium-on protocols were less effective. Li and Wang demonstrated that standard epithelium-off 
protocols were more effective at reducing maximum corneal curvature than epithelium-on protocols (Li and Wang, 2017), while Nath et al. reported that $7 \%$ of patients undergoing epithelium-on CXL experienced disease progression within the first year, compared to $2 \%$ of patients in the epithelium-off group $(p$-value $=0.022)$ (Nath et al., 2020). As a result, several modifications to epithelium-on CXL have been proposed.

\section{Chemical Enhancers}

Topical medications such as tetracaine, benzalkonium chloride, ethylenediaminetetraacetic acid and trometamol, which are toxic to the corneal epithelium, have been used to increase permeability of tight intraepithelial junctions and promote riboflavin diffusion through the epithelium (Cha et al., 2004). Meta-analysis of eight short-term 1 year studies of 455 keratoconus eyes showed that eyes treated with epithelium-on protocols and chemical enhancers experienced a comparable reduction in corneal curvature as eyes treated with standard epithelium-off protocol (Wen et al., 2018).

However, long-term results suggest that epithelium-on protocols with chemical enhancers alone are less effective compared to epithelium-off protocols. A 3 year comparative study of an epithelium-on protocol with chemical enhancers vs. standard epithelium-off patients established that although keratoconus progression was halted in both groups, epitheliumoff patients demonstrated superior corneal aberrometry and asphericity results (Arance-Gil et al., 2020).

\section{Iontophoresis-Assisted Riboflavin Delivery}

Iontophoresis has also been studied as an adjunct to improve riboflavin penetration in epithelium-on protocols. In iontophoresis-CXL, an electric field is created to increase diffusion of negatively charged riboflavin through the epithelium and stroma. A meta-analysis of 455 eyes with keratoconus by Wen et al. found that although comparable reduction in corneal curvature in eyes treated with standard epithelium-off protocol was achieved, patients treated with iontophoresis-assisted transepithelial protocols experienced lower reduction in corneal curvature compared to those treated with standard epithelium-off protocols (Wen et al., 2018).

\section{Phonophoresis-Assisted Riboflavin Delivery}

It is postulated that phonophoresis enhances pharmacological delivery via radiation forces, acoustic streaming, and acoustic cavitation (Pitt et al., 2004). An experimental procedure by Lamy et al. involved the use of ultrasound to augment the penetration of riboflavin into the corneal stroma over an intact epithelium. In this study, the authors utilised fluorescent riboflavin and an ultrasound device adjusted to produce continuous-wave ultrasound of $880 \mathrm{kHz}$ at $1 \mathrm{~W} / \mathrm{cm}^{2}$ to aid delivery of the drug. A statistically significant difference between riboflavin penetration was noted between non-ultrasound treated rabbit corneas and ultrasound treated rabbit corneas $(p<0.001)$. The authors concluded that ultrasound treatment aided entry of riboflavin into the corneal stroma despite the presence of an intact epithelial barrier (Lamy et al., 2013). At the same time, a mean increase in temperature of $6-7^{\circ} \mathrm{C}$ in eyes undergoing phonophoresis-assisted riboflavin delivery was identified. This raises safety concerns, as hyperthermia of $41^{\circ} \mathrm{C}$ and beyond has been associated with the development of cataracts and elevated corneal epithelium 70-kilodalton stress protein (Nabili et al., 2015). Further clinical studies are needed to further characterise the safety aspects of this method.

\section{Oral Riboflavin}

A novel approach to CXL without the need for epithelial debridement involves administration of oral riboflavin and natural sunlight exposure. In this proposed technique, patients ingest high doses of riboflavin and are exposed to 15 minutes of direct sunlight while engaging in daily exercise. A series of three cases of keratoconus treated in this manner described no adverse effects, with corneal flattening reported within 6 months of treatment (Jarstad et al., 2019). A small prospective study of 24 patients with keratoconus is underway to investigate the efficacy of high dose (400 mg) oral riboflavin (Jarstad et al., 2019).

Although oral riboflavin presents itself as an inexpensive and less invasive alternative to corneal cross-linking, its utility may be limited by the extended duration of treatment, variability in ultraviolet exposure and patient compliance to outdoor regimens. Additionally, given the long treatment duration and hence slower formation of cross-links, this method may not be optimal for patients with severe progressive keratoconus, which requires rapid stabilisation to prevent further progression. Limited data is also available regarding dose-response relationships of systemically absorbed riboflavin and its ocular bioavailability. Furthermore, the toxicity of systemic administration of high doses of riboflavin has yet to be wellestablished (Institute of Medicine US, 1998). The systemic administration of riboflavin may also function as an endogenous photosensitiser, which confers an increased risk of experiencing sunburn and photoaging of the skin (Pandel et al., 2013).

\section{Improving Oxygen Diffusion}

Oxygen is a key component of the Type 2 oxidative pathway in the reaction between riboflavin and UVA. However, in epithelium-on protocols, stromal oxygen diffusion is limited by the intact epithelium. The corneal epithelium absorbs almost ten times the amount of oxygen that the corneal stroma does (Freeman, 1972). Therefore, riboflavin's crosslinking efficacy in epithelium-on protocols is reduced within an oxygen-poor stromal environment, (Richoz et al., 2013). To overcome this, several solutions have been proposed.

\section{Pulsed Ultraviolet-A}

Previous studies have shown that continuous high UVA irradiation results in unsatisfactory riboflavin-induced corneal stiffening due to inadequate oxygen diffusion (Peyman et al., 2016; Choi et al., 2017; Kirgiz et al., 2019). Hence, it was suggested that pulsed fractionation of UVA irradiation may improve crosslinking efficacy by allowing re-diffusion of oxygen during pauses in between UVA light pulses (Richoz et al., 2013).

This postulation was confirmed by a randomised controlled trial of 60 patients conducted by Moramarco et al. which 
compared accelerated CXL using continuous UVA exposure at $30 \mathrm{~mW} / \mathrm{cm}^{2}$ for 4 minutes with accelerated CXL using pulsed UVA with 8 minutes (1 second on: 1 second off) of UVA exposure at $30 \mathrm{~mW} / \mathrm{cm}^{2}$. Their study showed that the stromal demarcation line was significantly deeper in the pulsed UVA group $(213 \pm 47.38 \mu \mathrm{m})$ compared to the continuous UVA group $(149.32 \pm 36.03 \mu \mathrm{m})$ (Moramarco et al., 2015). Another similar randomised controlled trial of 70 eyes conducted by Peyman et al. corroborated these findings, with a significantly deeper stromal demarcation line observed in the pulsed group compared to the continuous group $(201.11 \pm 27.76$ vs $159.88 \pm 20.86 \mu \mathrm{m})$ (Peyman et al., 2016). However, under laboratory settings, pulsed ultraviolet-A light alone did not substantially improve the increase in corneal biomechanical strength (Kling et al., 2015).

Gore et al. studied an accelerated pulsed high-fluence protocol for progressive keratoconus on 756 eyes (Gore et al., 2021). Corneas with thickness $<375 \mu \mathrm{m}$ were excluded. High-fluence, pulsed ultraviolet-A was delivered at $30 \mathrm{~mW} / \mathrm{cm}^{2}$ for 4 minutes with a $1.5 \mathrm{~s}$ on/off cycle. The total energy delivered was $7.2 \mathrm{~J} / \mathrm{cm}^{2}$. At 6 and 12 months, the study team noted modest corneal flattening with keratometric stabilisation in $98.3 \%$ of eyes. No changes in central keratometry were noted. Moreover, mean corrected distance visual acuity, manifest refraction and endothelial cell density did not change.

\section{Enhanced-Fluence Pulsed-Light lontophoresis Cross-Linking}

Due to its improved efficacy, pulsed UVA irradiation was studied as an adjunct to the iontophoresis-assisted epithelium-on protocol to improve cross-linking efficiency. Mazzotta et al. conducted a prospective interventional pilot study of 24 eyes of 20 patients with keratoconus. Patients underwent iontophoresis-assisted cross-linking with riboflavin solution and received UVA irradiation of $18 \mathrm{~mW} / \mathrm{cm}^{2}$ of pulsed-light on-off exposure. This combination of increased riboflavin transport and oxygen availability across the epithelium produced favourable results.

At 3 years, the average uncorrected distance visual acuity improved from $0.50 \pm 0.10$ to $0.36 \pm 0.08 \log$ MAR and average maximum keratometry readings decreased from $52.94 \pm 1.34$ to $51.4 \pm 1.49$ diopters. Additionally, anterior segment optical coherence tomography showed that the demarcation lines were situated at an average depth of $285.8 \pm 20.2 \mu \mathrm{m}$ in more than $80 \%$ of patients at 1 month postoperatively (Mazzotta et al., 2020a). This shows that with pulsed UVA irradiation, epitheliumon cross-linking can achieve a demarcation line depth that is close to that of one created by the standard epithelium-off crosslinking. Further comparative clinical studies with epitheliumoff protocols are warranted.

\section{Oxygen Supplementation}

Besides pulsed UVA, oxygen supplementation of the corneal surface has been shown to increase the strength and depth of CXL (Seiler et al., 2020). A wearable oxygen delivery device, known as Boost (Avedro, MA, United States), is a recent approach to epithelium-on corneal cross-linking. It creates a hyperoxic periocular environment. Ex-vivo studies have shown that periocular oxygen supplementation provides significantly more corneal stiffening (Adler et al., 2019). A randomised, agesex-matched study by Aydin et al. demonstrated that patients treated with periocular oxygen supplemented accelerated epithelium-on protocol experienced a larger decrease in maximum keratometry values $(p$-value $=0.019)$ and had a significantly deeper demarcation line $(320 \pm 17 \mu \mathrm{m})$ when compared to the control group $(269 \pm 19 \mu \mathrm{m})$ (Aydin and Aslan, 2021). Additionally, post-procedural endothelial cell density was comparable between both groups.

\section{Improving Visual Acuity}

Although epithelium-off riboflavin and UVA cross-linking is highly effective in halting the progression of keratoconus, it provides limited improvement in visual acuity (Raiskup and Spoerl, 2013; Wittig-Silva et al., 2014; Seyedian et al., 2015; Taşç et al., 2020). As a result, several adjunct devices and procedures have been used in combination with riboflavin administration to improve visual outcomes.

\section{Photorefractive Keratectomy With Corneal Cross-Linking}

The Athens protocol involves performing topographically-guided transepithelial photorefractive keratectomy (PRK) followed by CXL. Early studies have shown that a combination of PRK with CXL offers keratoconic eyes better visual acuity and corneal stability (Kymionis et al., 2009). A recent study by Kontadakis et al. compared simultaneous topography-guided PRK and CXL (tPRK-CXL group) with CXL alone and found that uncorrected distance visual acuity at 3 years was significantly better in the tPRK-CXL group $(0.27 \pm 0.25 \log M A R)$ as compared to the CXLonly group $(0.69 \pm 0.58 \log \mathrm{MAR})$. Moreover, steep and flat keratometric readings were also flatter in the tPRK-CXL group (Kontadakis et al., 2016).

Kanellopoulos et al. further proposed the enhanced Athens protocol, incorporating a customised and fluence topography-guided UVA irradiation to maximise refractive normalisation of the cornea with lesser stromal tissue removal than the standard Athens protocol (Kanellopoulos, 2019). Finally, Minneapolis' epi-off protocol, which utilises simultaneous CXL with Phorcides analytical software topography-guided correction photorefractive keratectomy is also being explored as a treatment modality (Hammond and Lobanoff, 2019).

\section{Transepithelial Phototherapeutic Keratectomy With Corneal Cross-Linking}

The Cretan protocol involves performing transepithelial phototherapeutic keratectomy (tPTK) with corneal crosslinking. In 2010, Kymionis et al. reported a case of keratoconus treated with the Cretan protocol. This patient achieved an improvement in postoperative uncorrected visual acuity and best spectacle-corrected visual acuity with stabilisation of keratoconus progression (Kymionis et al., 2010). A clinical comparative study of the Cretan and Dresden protocol subsequently showed that the mean uncorrected distance visual acuity and corrected distance visual acuity of eyes 
treated with the Cretan protocol improved from logMAR $0.99 \pm$ 0.71 and $0.30 \pm 0.26$ preoperatively to $0.63 \pm 0.42$ and $0.19 \pm 0.18$ at 12 months postoperatively, respectively. Neither uncorrected nor corrected distance visual acuity demonstrated any significant improvement postoperatively, and at 12 months in the Dresden protocol group (Kymionis et al., 2012a). A 3 year prospective comparative study of 30 eyes conducted by Grentzelos et al. showed that not only did uncorrected and corrected distance visual acuity improve up to 3 year postoperatively, mean corneal astigmatism was also reduced from $-6.19 \pm 4.54$ diopters preoperatively to $-4.68 \pm$ 3.10 diopters. Patients who underwent routine epithelium-off CXL did not experience improvements in visual acuity or corneal astigmatism (Grentzelos et al., 2019).

\section{Intrastromal Corneal Ring Segment Implantation With Corneal Cross-Linking}

Intrastromal corneal ring segment implantation has been studied as a treatment modality for corneal ectasias. Alió et al. studied the effects of intrastromal corneal ring segment implantation in eyes with keratoconus, while Kymionis et al. followed up on eyes with post-LASIK ectasia (Alió et al., 2006; Kymionis et al., 2006). Both studies showed that long-term refractive stability was achieved. However, keratometry values worsened after 36 months amongst eyes with keratoconus (Alió et al., 2006). Kim et al. subsequently reported that intracorneal ring segment implantation followed by CXL with riboflavin within 1 month had a greater effect on improvements in visual acuity and reduction in refractive and keratometric values compared to intracorneal ring segment implantation or CXL alone (Kim and Kim, 2019).

Singal et al. conducted a large prospective study of 542 eyes comparing CXL alone ( $n=204)$ (CXL-alone), CXL with intracorneal ring segment implantation $(n=126)$ (CXL-ICRS) and topography-guided photorefractive keratectomy with CXL $(n=122)$ (CXL-TG-PRK) in patients with progressive keratoconus, pellucid marginal degeneration, or LASIKinduced ectasia. It was noted that changes in uncorrected distance visual acuity was significant in patients undergoing CXL-ICRS $(-0.31 ; 95 \% \mathrm{CI},-0.38$ to -0.24$)$ and CXL-TG-PRK ( -0.16 ; $95 \% \mathrm{CI},-0.24$ to -0.09$)$, but not in CXL-only. Moreover, changes in maximum keratometry value were significant amongst eyes which underwent CXL-ICRS (-3.21 diopters (D); $95 \% \mathrm{CI},-3.98$ to -2.45$)$ and CXL-TG-PRK $(-3.69 \mathrm{D}$; $95 \% \mathrm{CI},-4.49$ to -2.90$)$, but not with CXL alone $(-0.05 \mathrm{D}$; $95 \% \mathrm{CI},-0.66$ to 0.55 ) (Singal et al., 2020). The authors concluded that CXL with intrastromal corneal ring segment implantation may be more effective for eyes with greater irregular astigmatism and worse visual acuity, while topography-guided photorefractive keratectomy with CXL is effective for eyes requiring improvement in irregular astigmatism but still with good corrected distance visual acuity.

\section{Customised Remodelled Vision (CurV) Protocol}

Excitingly, the new Customised Remodelled Vision (CurV) protocol can improve the quality of vision by individualising
UVA irradiation patterns guided by corneal topography (Figure 2). It is theorised that corneal biomechanical weakness in keratoconus is focal in nature (Roberts and Dupps, 2014). Hence, in contrast to the generalised UVA irradiation administered with the Dresden protocol, CurV irradiates ectatic parts of the cornea with more intense UVA irradiation whilst stronger areas are treated with little or no UVA. One year results by Seiler et al. were encouraging, with patients treated with CurV showing greater changes in maximum keratometry values $(-1.7 \pm 2.0$ diopter vs. $-0.9 \pm$ 1.3 diopter) and superior epithelial healing time (2.56 \pm 0.50 days vs. $3.19 \pm 0.73$ days) than patients treated with an epithelium-off protocol (Seiler et al., 2016). These findings are further supported by a study performed by Cassagne et al., who found that CurV induced a greater change in maximum keratometry values compared to standard epithelium-off protocol and improved corrected distance visual acuity $(0.2162 \pm 0.2495 \operatorname{logMAR}$ vs $0.2648 \pm 0.2574 \log$ MAR) over a follow-up duration of a year (Cassagne et al., 2017).

Mazzotta et al. recently investigated the utility of highirradiance epithelium-on customised CXL with supplemental oxygen (Mazzotta et al., 2020b). A total of 27 eyes were included in this study. Patients experienced significant improvement in corrected distance visual acuity with flattening of steep keratometry (mean change of -1.9 dioptres, $p$-value $<0.05)$ at their follow-up 6 months after the procedure. Additionally, two demarcation lines were observed at mean depths of $218.23 \pm 43.32 \mu \mathrm{m}$ and $325.71 \pm 39.70 \mu \mathrm{m}$. Notably, these demarcation lines were approximately $30 \%$ deeper in this series than previously reported by studies which utilised epithelium-off CurV protocols. This suggests the possibility of conducting CurV without the need for de-epithelisation. More long-term comparative studies are warranted to determine the efficacy of CurV.

\section{ANTI-INFECTIVE APPLICATION OF RIBOFLAVIN AND ULTRAVIOLET-A FOR THE TREATMENT OF INFECTIVE KERATITIS (PACK-CXL)}

CXL has been studied as an alternative to antibiotics in the treatment of infectious keratitis. Termed photoactivated chromophore for infectious keratitis - corneal crosslinking (PACK-CXL), it has been suggested to provide added resistance to pepsin and collagenase enzymes produced by bacterial and fungal pathogens (Hafezi and Randleman, 2014; Vajpayee et al., 2015). Additionally, UVA and riboflavin have been postulated to confer synergistic antimicrobial effects. UVA damages microbial DNA and RNA; thereby inhibiting microbial replication, while photoactivated riboflavin releases free radicals that damage cell membranes and nucleic acids (Vazirani and Vaddavalli, 2013). 


\section{Bacterial Keratitis}

Recent studies have shown that PACK-CXL offers superior efficacy and reduces duration of healing of bacterial keratitis. Tawfeek et al. randomised 78 eyes with bacterial keratitis into a control group receiving appropriate topical conventional broad-spectrum antimicrobial therapy alone and a group with topical antibiotics with adjuvant PACK-CXL. It was noted that a higher proportion of patients in the group receiving combination treatment of antibiotics with PACK-CXL experienced complete resolution of ulcers (97.4 vs $76.9 \%, p$-value $<0.001)$. Additionally, the mean resolution period was shorter in patients treated with PACK-CXL (7.02 \pm 2 weeks vs $10.87 \pm 3.28$ weeks, $p$-value $=0.002)$ (Tawfeek et al., 2020).

Knyazer and colleagues have reported that additional PACKCXL significantly reduces time to epithelial healing in ulcers up to $4 \mathrm{~mm}$ in size when using accelerated PACK-CXL and standardfluence of $5.4 \mathrm{~J} / \mathrm{cm}^{2}$ (Knyazer et al., 2020). A recently performed prospective randomized Swiss multicenter PACK-CXL trial further demonstrated that PACK-CXL alone is as efficient as antimicrobial therapy in small ulcers up to $4 \mathrm{~mm}$ in size (TorresNetto et al., 2020).

In patients with treatment-resistant post-penetrating keratoplasty infectious keratitis, PACK-CXL in comparison to medical therapy alone, offered greater rates of resolution (83.3 vs $68.2 \%, p$-value $=0.28$ ), healing duration (average of 24 vs 34 days, $p$-value $=0.02)$ and reduction in graft failure rates $(27.8$ vs $54.5 \%$, $p$-value $=0.08)$ in the PACK-CXL group, although some of these results were not statistically significant (Ozbek-Uzman et al., 2020).

\section{Fungal Keratitis}

In contrast, PACK-CXL has been reported to be associated with increased risk of corneal perforation and visual acuity deterioration when used to treat fungal keratitis (Uddaraju et al., 2015; Prajna et al., 2020). A randomised control trial of 111 patients found no difference in culture positivity at 24 hours, re-epithelisation rates or scar size between those randomised to anti-fungals alone compared to PACK-CXL with antifungals (Prajna et al., 2020). Moreover, the best spectacle-corrected visual acuity at 3 weeks and 3 months appeared to deteriorate in the PACK-CXL group. However, concerns were raised from two different groups regarding the methodology of the study (Ting et al., 2020; Hafezi et al., 2021b). On the other hand, Wei et al. randomized 41 patients with fungal keratitis into two groups-one group was treated both CXL and antifungals, while patients in the other group were treated with antifungals only. They demonstrated that CXL in combination with antifungals accelerated the duration of ulcer healing, reduced the frequency of administered medications, and significantly reduced the maximum ulcer depth after treatment (Wei et al., 2019). Rose Bengal Photodynamic Antimicrobial Therapy (RBPDAT) has been studied as an alternative, and a pilot study of 18 eyes showed that $72 \%$ of the treated eyes avoided the need for therapeutic penetrating keratoplasty (Naranjo et al., 2019).

\section{REDUCING RIBOFLAVIN/ULTRAVIOLET-A TOXICITY FOR THE TREATMENT OF THIN CORNEAS}

\section{Toxicology Profile of Riboflavin and Ultraviolet-A}

Ultraviolet rays have the propensity to induce structural damage depending on its wavelength, intensity, and irradiation duration. Direct exposure to ultraviolet rays may induce photokeratitis, endothelial damage, cataracts, and even retinal injury. However, ultraviolet-A-induced damage is unlikely to occur in CXL because riboflavin shields these structures from potential injury (Spoerl et al., 2007). The degree of ultraviolet-A shielding by riboflavin is believed to be governed by the Beer-Lambart Law, which states a linear relationship between the concentration and absorbance of the solution (riboflavin) (Spoerl et al., 2007; Iseli et al., 2011). Experimentally, this relationship holds true for lower concentrations of riboflavin - the absorbance coefficient of riboflavin solutions increases linearly up to a concentration of $0.04 \%$, before plateauing and remaining constant for higher concentrations (Spoerl et al., 2007). Hence, injury secondary to direct ultraviolet-A irradiation is unlikely to occur in a clinical setting where $0.1 \%$ riboflavin is used.

However, Wollensak et al. reported that photopolymerisation between riboflavin and ultraviolet-A produces cytotoxic free radicals which can cause significant damage, especially to the corneal keratocytes and endothelial cells (Wollensak et al., 2003b; 2004b; 2004a). Furthermore, Caporossi et al. showed that of 10 eyes treated with riboflavin/ultraviolet-A clinically, keratocyte apoptosis occurred up to depths ranging from 270 to $350 \mu \mathrm{m}$. Fortunately, such damage appears to be transient as repopulation of keratocytes with a normal keratocyte density within the entire corneal stroma was observed at 6 months (Caporossi et al., 2006).

Conversely, endothelial cell damage after CXL resulting in severe corneal oedema that impedes visual acuity have been reported in literature (Bagga et al., 2012; Sharma et al., 2012). Wollensak et al. demonstrated that ultraviolet-A irradiance of $0.35 \mathrm{~mW} / \mathrm{cm}^{2}$ causes endothelial cell toxicity at depths greater than $400 \mu \mathrm{m}$ (Wollensak et al., 2003b). Moreover, Kymionis et al. reported a significant decrease of endothelial cell density (mean decrease of 292 cells $/ \mathrm{mm}^{2}$ ) after performing standard epitheliumoff corneal crosslinking in 14 eyes with thin corneas (range

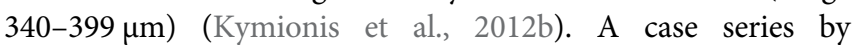
Sharma et al. identified 10 patients out of a cohort of 350 patients with persistent post-CXL corneal oedema, which was postulated to have occurred from inadvertent corneal endothelial damage (Sharma et al., 2012). Therefore, a minimum corneal thickness of $400 \mu \mathrm{m}$ was previously suggested as a prerequisite for epithelium-off CXL due to the risk of endothelial cell toxicity (Sharma et al., 2012)

Given the above-mentioned risk of endothelial cell toxicity, a potential challenge in CXL is treating patients with severe disease where their corneal thickness is less than $400 \mu \mathrm{m}$. To overcome this, multiple solutions such as altering the osmolarity of riboflavin formulation, using assistive techniques to change riboflavin delivery and customising UVA irradiation to induce 
riboflavin cross-link formations at varying depths have been proposed.

\section{Hypoosmolar Riboflavin}

Hypoosmolar riboflavin in a dextran-free solution has been used to induce stromal swelling and consequently increase corneal thickness in patients with thin corneas. Corneal swelling occurs due to hydrophilic properties of stromal proteoglycans, which form "collagen-free lakes" and increases corneal thickness (Hafezi et al., 2009). Several studies have shown that hypoosmolar CXL formulations for thin corneas aids in the stabilisation of keratoconus with no resulting endothelial cell loss (Hafezi et al., 2009; Raiskup and Spoerl, 2011). However, the efficacy of hypoosmolar riboflavin has been suggested to be less pronounced compared with standard formulations (Stojanovic et al., 2014). This may occur due to reduced concentrations of collagen fibrils and poor oxygen diffusion secondary to increased hydration of the corneal stroma. Additionally, Hafezi et al. have reported that hypoosmolar riboflavin results in large interindividual variations in corneal swelling duration and range (swelling duration ranged from 3 to 20 minutes extent of swelling ranged from 36 to $105 \mu \mathrm{m}$ ) (Hafezi et al., 2009).

\section{Contact-Lens Assisted Corneal Cross-Linking (CACXL)}

In 2014, Jacob et al. described a novel method of inducing corneal swelling in patients with keratoconus and thin corneas measuring between $350 \mu \mathrm{m}$ and $400 \mu \mathrm{m}$. The proposed protocol involved placing an ultraviolet barrier-free soft contact lens $(0.09-\mathrm{mm}$ thickness, $14-\mathrm{mm}$ diameter) soaked in iso-osmolar riboflavin $0.1 \%$ for 30 minutes on the cornea. Once the corneal thickness was confirmed to be greater than $400 \mu \mathrm{m}$, UVA irradiation was commenced combined with administration of iso-osmolar riboflavin $0.1 \%$. Their study found that CACXL achieved a stromal demarcation line mean depth of $252.9 \pm 40.8 \mu \mathrm{m}$, with no significant endothelial loss secondary to UVA toxicity identified (Jacob et al., 2014). However, the presence of a contact lens over the epithelium creates an artificial barrier that reduces oxygen diffusion into the stroma, which reduces efficacy of riboflavin and UVA in inducing cross-linking (Kling et al., 2017; Wollensak et al., 2019).

\section{Epithelial Island Cross-Linking Technique}

Another potential treatment which adjusts the way riboflavin is applied to the corneal surface is customised pachymetry guided epithelial debridement. This technique requires epithelial debridement over areas of thicker cornea, while leaving an "island" of undebrided epithelium over the thinner apical area. Once riboflavin is applied, the epithelial island protects the thin apical cornea from UVA. Mazzotta et al. followed-up a small series of patients who underwent this technique and found it to be safe and efficacious (Mazzotta and Ramovecchi, 2014). Cagil et al. subsequently performed this technique on 19 eyes and observed an improvement in uncorrected visual acuity, best corrected visual acuity, flattest keratometry value and steepest keratometry values 1 year postoperatively. However, there was significant endothelial cell density loss (2550 \pm 324 vs $2030 \pm 200$ cells $/ \mathrm{mm}^{2}$ ) 1 year postoperatively (Cagil et al., 2017).

\section{Epi-Off-Lenticule-on Corneal Cross-linking}

Sachdev et al. have reported three cases of successful CXL performed on thin, de-epithelialised corneas overlaid with a donor corneal lenticule. The refractive lenticules were obtained from patients who had undergone small incision lenticule extraction (SMILE). Instead of being applied directly onto the debrided corneal epithelium, this protocol requires riboflavin to be applied on a donor corneal lenticule. In this case series, this technique was found to be safe and effective (Sachdev et al., 2015). A study by Cagini et al. of this technique showed that visual acuity and endothelial cell density remained stable over a 12 month followup duration. Moreover, the presence of a demarcation line was seen in all patients by 6 months of follow-up (Cagini et al., 2020).

\section{Pachymetry-Based Accelerated Cross-Linking}

Mazzotta et al. have proposed the M normogram, which puts together all available high-quality evidence on the depth of the demarcation line achieved across varying cross-linking protocols. This allows surgeons to choose from a list of protocols (of varying UVA regime and riboflavin formulation) to achieve the desired depth of cross-linking to minimise the risk of inducing endothelial cell toxicity. The $M$ nomogram was validated against clinical findings of 20 eyes. Of these, eyes treated with $3 \mathrm{~mW} / \mathrm{cm}^{2}$ conventional protocol showed an average demarcation depth of $350 \pm 50 \mu \mathrm{m}$, while eyes treated with $30 \mathrm{~mW} / \mathrm{cm}^{2}$ continuous light accelerated CXL had demarcation depths of $200 \pm 50 \mu \mathrm{m}$. Additionally, eyes treated with $30 \mathrm{~mW} / \mathrm{cm}^{2}$ pulsed light accelerated CXL had depths at $250 \pm 50 \mu \mathrm{m}$ while eyes treated with $15 \mathrm{~mW} / \mathrm{cm}^{2}$ pulsed light accelerated CXL had depths at $280 \pm 30 \mu \mathrm{m}$. Comparison of the measured demarcation line depths showed a high correlation between the measured and calculated depth based on the $M$ nomogram ( $\mathrm{m}=1.03, R^{2}=0.73$ ) (Mazzotta et al., 2018). However, this protocol has a distinct limitation - it requires surgeons to have access to various riboflavin formulations, and cross-linking devices that can output UVA energy at 3,9, 15, and even $30 \mathrm{~mW} / \mathrm{cm}^{2}$; using either continuous light or pulsed light protocols. Moreover, iontophoresis may also be required in some cases to perform the treatment.

\section{Sub400 Protocol}

A study by Hafezi et al. introduced the sub400 individualised fluence CXL protocol which allows CXL to be performed on corneas with thickness of less than $400 \mu \mathrm{m}$ (Hafezi et al., 2020). This aimed to circumvent disadvantages of reduced cross-linking efficacy conferred by earlier approaches that induced corneal swelling through hypoosmolar riboflavin or contact lens-assisted corneal cross-linking, and the need for specialised equipment for techniques such epi-Off-Lenticule-on and pachymetry based corneal cross-linking (Stojanovic et al., 2014).

Instead of modifying cornea thickness or riboflavin formulation, the sub400 protocol adjusts the UV illumination 
time and irradiance according to the corneal thickness to achieve a safe depth of cross-linking $70 \mu \mathrm{m}$ away from the endothelium. The algorithm was published in 2017 and based on this algorithm, a pilot study of 39 eyes with corneal stromal thickness ranging from $214 \mu \mathrm{m}$ to $398 \mu \mathrm{m}$ showed that $90 \%$ of eyes established topographical stability at 12 months, while no eyes experienced endothelial decompensation (Kling and Hafezi, 2017). There was a significant improvement in corneal maximum keratometry values $(-2.06 \pm 3.66$ diopters $)$, but there were no changes in corrected distance visual acuity (Hafezi et al., 2020). This individualised approach is a promising new addition to existing treatment modalities for thin corneas, especially since this can be accomplished using standard CXL equipment.

\section{AND PACK-CXL AT THE SLIT-LAMP (C-EYE DEVICE)}

Conventionally, PACK-CXL must be performed by corneal surgeons in a sterile operating theatre. C-Eye (EMAGine, Switzerland) is a miniaturised UVA irradiation system that can be mounted on a slit-lamp and operated by ophthalmologists in an ophthalmic clinic (Hafezi et al., 2021a). This device may provide better patient comfort because it allows patients to remain seated in front of the slit-lamp during the procedure. Epithelial debridement is performed by applying $40 \%$ ethanol over the central epithelium. Following which, riboflavin solution is applied, and UVA irradiation is administered. An exvivo study has demonstrated that this upright position that the patient adopts during UVA irradiation does not affect riboflavin distribution, with gravitational influence on riboflavin distribution only observed after 60 minutes of vertical positioning, well above the requirements of cross-linking which typically takes between 3 and 30 minutes (Salmon et al., 2017). The C-Eye device can also be used to perform cross-linking procedures for various corneal ectasias. This device may allow PACK-CXL to be better integrated as a treatment for infective keratitis during clinical care and allow PACK-CXL to be conducted even in resource-limited settings.

\section{OTHER INDICATIONS FOR CORNEAL CROSS-LINKING}

\section{Combined Refractive Surgery With Corneal Cross-Linking (LASIK Xtra and SMILE Xtra)}

Laser in situ keratomileusis (LASIK) and small incision lenticule extraction (SMILE) are procedures utilised to treat refractive errors. Simultaneous use of CXL with SMILE or LASIK in the same sitting is termed LASIK Xtra or SMILE Xtra and is postulated to strengthen the cornea postoperatively and reduce the development of postoperative corneal ectasia.

A study examining LASIK Xtra and SMILE Xtra protocols in LASIK rabbit ectasia models showed that mean curvature values decreased significantly following treatment. Furthermore, mean posterior elevation post-LASIK Xtra was found to be reduced compared to conventional LASIK (Konstantopoulos et al., 2019). While safe, two prospective randomised-controlled trials of LASIK Xtra vs. conventional LASIK have shown variable results. A study by Kanellopoulos et al. observed improved refractory and keratometric stability after LASIK Xtra (Kanellopoulos and Asimellis, 2015), while a study by Kohnen et al. showed no significant advantage in visual acuity, refraction or keratometric measurements with LASIK Xtra at 1 year followup (Kohnen et al., 2020). Liu et al. examined patients who underwent SMILE Xtra and found that its safety, efficacy, predictability, and stability are comparable with those of Femtosecond-LASIK Xtra at 1 year follow-up (Liu et al., 2021).

\section{Hyper-osmolar Riboflavin for Treatment of Bullous Keratopathy}

CXL has been described in the management of patients with bullous keratopathy. Modifications to the riboflavin formulation includes use of intraoperative glycerol $70 \%$ or preoperative use of glucose $40 \%$ to dehydrate and reduce corneal oedema in patients with bullous keratopathy (Wollensak et al., 2009; Hafezi et al., 2010). In both studies, a reduction in central corneal thickness and best corrected visual acuity was observed, with no further episodes of bullae rupture and no re-intervention required postoperatively.

\section{CONTRAINDICATIONS AND COMPLICATIONS}

\section{Contraindications to Corneal Cross-linking}

Several contraindications for CXL have been suggested. These include pregnant or breastfeeding patients, patients with prior herpetic infections (due to the risk of herpes simplex virus reactivation), active ocular inflammation, severe central or paracentral corneal scars, autoimmune disorders, and a history of poor epithelial wound healing. Corneal thickness of less than $400 \mu \mathrm{m}$ is increasingly considered a relative contraindication as it can be circumvented by utilising various techiniques described above.

\section{Transient Corneal Haze}

Transient corneal haze after CXL is not uncommon. A study by Koller and colleagues prospectively evaluated 117 eyes which had undergone CXL and found that all eyes had anterior stromal haze at 1 month which subsequently improved within a 1 year period (Koller et al., 2009). Another randomised control trial of 36 patients with keratoconus found that the mean densitometry peaked at 1 month after treatment, but subsequently returned to baseline values after 6 months (Kim et al., 2016). Transient corneal haze is postulated to occur secondary to increased collagen fibre diameter post-CXL (Kozobolis et al., 2016). This is not typically associated with visual disturbances and often regresses after 12 months. 


\section{Persistent Corneal Haze}

On the other hand, persistent corneal haze lasting beyond 12 months should be differentiated from transient haze as this can negatively impact visual acuity and may respond to intensive topical steroid treatment (Mazzotta et al., 2015). This is thought to be secondary to the ongoing keratectasia process and corneal remodelling. A cohort study consisting of 34 eyes performed by Raiskup et al. observed 13 eyes (38.3\%) with persistent corneal haze over a follow-up period of 10 years. (Raiskup et al., 2015). Preoperative risk factors identified include age over 35 years advanced keratoconus with minimal corneal thickness $<400 \mu \mathrm{m}$ and presence of preoperative activated keratocytes in the anterior stroma when measured with confocal microscopy. Intraoperative risk factors include forward defocus of UVA source, lack of riboflavin $0.1 \%$ application during irradiation and excessive riboflavin-dextran $20 \%$ solution causing stromal dehydration. Postoperative risk factors reported include noncompliance with postoperative topical corticosteroid therapy, infective keratitis, therapeutic lens intolerance, and presence of Langerhan cells after contact lens removal postoperatively (Mazzotta and Caragiuli, 2014; Mazzotta et al., 2015; Kim et al., 2016).

\section{Infectious Keratitis}

Multiple incidences of post-CXL infectious keratitis have been reported. Offending organisms included Gram-negative organisms (escherichia coli, pseudomonas aeruginosa), Grampositive organisms (staphylococcus aureus, streptococcus species), fungi and herpes simplex virus (Pollhammer and Cursiefen, 2009; Sharma et al., 2010; Al-Amry et al., 2017; Sitaula et al., 2019; Kodavoor et al., 2020). A recent study by Tzamalis and colleagues showed that postoperative application of bandage contact lens and topical steroids were independent risk factors for the development of microbial keratitis (Tzamalis et al., 2019).

The precise mechanism for herpes simplex virus reactivation after CXL remains unknown. It is postulated that emotional stress, trauma, and exposure to UVA are possible mechanisms of reactivation (Evangelista and Hatch, 2018).

\section{Other Complications}

Further risks associated with CXL vary depending on the protocol used. In addition to corneal haze and post-CXL infectious keratitis, other complications include the development of a persistent epithelial defect, stromal scarring, corneal melt, corneal endothelial decompensation and damage to the lens or retina (Wollensak et al., 2003a). Development of late onset peripheral ulcerative keratitis following CXL has also been reported (Chanbour et al., 2019).

\section{FUTURE DEVELOPMENTS}

A few areas are of particular interest. First, the dose-response relationship of UVA irradiation and riboflavin remains unclear. Although mathematical models have been postulated, their accuracy remains controversial as the exact molecular interactions of CXL remain elusive. Understanding the relationship between UVA, riboflavin and oxygen availability will enable clinicians to modify protocols in an informed and safe manner.

Second, in complementing the increasing utility of CXL as an anterior segment therapy, diagnostic tools are continually proposed to pick up subclinical ectasias. Corneal epithelial mapping is a procedure that is currently in vogue. It measures corneal epithelial and stromal thickness to identify areas of hypertrophy or thinning associated with corneal ectasias ( $\mathrm{Li}$ et al., 2016). Machine learning algorithms which identify subclinical keratoconus have also been developed based on these measurements and are currently undergoing refinement (Shi et al., 2020). This will be useful in the identification and monitoring of progression, which may assist ophthalmologists in formulating recommendations for early corneal cross-linking.

Finally, indications for riboflavin as a photosensitiser for cross-linking have expanded beyond corneal ectasias. It is currently studied for use in correcting low myopia; and among post-radial keratotomy patients to reduce diurnal visual fluctuations (Elbaz et al., 2014; Elling et al., 2018).

\section{CONCLUSION}

Advancements in CXL have changed the way we approach and manage keratectasias and a range of corneal diseases. The molecular mechanisms of CXL and its impact on corneal biomechanics continue to be extensively studied, with the hope that improvements can be made that enhance the efficacy and safety of this treatment. The advent of individualised UVA irradiation patterns may pave the way for safer and targeted treatment for keratoconus in the near future. Larger, long-term studies are required to validate the efficacy of new CXL techniques, but with the rapid accumulation of knowledge and experience in the field, CXL has become a robust treatment option for corneal ectasias, and is well primed to become a useful addition to our armamentarium for treating infectious keratitis and potentially in the future, even small refractive errors.

\section{AUTHOR CONTRIBUTIONS}

CL and RM conceived the original idea of the review. DW and CL led and drafted the manuscript. DK-AL, BL, NW, FH, and RM reviewed the manuscript and provided critical feedback.

\section{FUNDING}

Publication fees associated with this article were funded by the Department of Ophthalmology, National University Hospital, Singapore. The authors would like to thank Dr. Mark Hillen for his valuable assistance in reviewing and revising the manuscript. We would also like to thank the Ophthalmology department of the National University Hospital in Singapore for supporting this review. 


\section{REFERENCES}

Abahussin, M., Hayes, S., Knox Cartwright, N. E., Kamma-Lorger, C. S., Khan, Y., Marshall, J., et al. (2009). 3D Collagen Orientation Study of the Human Cornea Using X-ray Diffraction and Femtosecond Laser Technology. Invest. Ophthalmol. Vis. Sci. 50, 5159-5164. doi:10.1167/iovs.09-3669

Adler, D. C., Hill, J., Liu, C., Deardorff, P., Raizman, M., and Rajpal, R. (2019). Biomechanical Impact of Drug Formulation, Supplemental Oxygen, and UV Delivery on Epi-On CXL. Invest. Ophthalmol. Vis. Sci. 60, 317.

Al-Amry, M., Mudhaiyan, T., Al-Huthail, R., and Al-Ghadeer, H. (2017). Infectious Crystalline Keratopathy After Corneal Cross-Linking. Middle East. Afr. J. Ophthalmol. 24, 100-102. doi:10.4103/meajo.MEAJO_346_16

Alió, J. L., Shabayek, M. H., and Artola, A. (2006). Intracorneal Ring Segments for Keratoconus Correction: Long-Term Follow-Up. J. Cataract Refract. Surg. 32, 978-985. doi:10.1016/j.jcrs.2006.02.044

Arance-Gil, Á., Villa-Collar, C., Pérez-Sanchez, B., Carracedo, G., and GutiérrezOrtega, R. (2021). Epithelium-Off vs. Transepithelial Corneal Collagen Crosslinking in Progressive Keratoconus: 3 Years of Follow-Up. J. Optom. 14, 189-198. doi:10.1016/j.optom.2020.07.005

Aydın, E., and Aslan, M. G. (2021). The Efficiency and Safety of OxygenSupplemented Accelerated Transepithelial Corneal Cross-Linking. Int. Ophthalmol. [Epub ahead of print]. doi:10.1007/s10792-021-01859-1

Bagga, B., Pahuja, S., Murthy, S., and Sangwan, V. S. (2012). Endothelial Failure After Collagen Cross-Linking with Riboflavin and UV-A. Cornea 31, 1197-1200. doi:10.1097/ICO.0b013e31823cbeb1

Brummer, G., Littlechild, S., McCall, S., Zhang, Y., and Conrad, G. W. (2011). The Role of Nonenzymatic Glycation and Carbonyls in Collagen Cross-Linking for the Treatment of Keratoconus. Invest. Ophthalmol. Vis. Sci. 52, 6363-6369. doi:10.1167/iovs.11-7585

Cagil, N., Sarac, O., Can, G. D., Akcay, E., and Can, M. E. (2017). Outcomes of Corneal Collagen Crosslinking Using a Customized Epithelial Debridement Technique in Keratoconic Eyes with Thin Corneas. Int. Ophthalmol. 37, 103-109. doi:10.1007/s10792-016-0234-3

Cagini, C., Riccitelli, F., Messina, M., Piccinelli, F., Torroni, G., Said, D., et al. (2020). Epi-off-lenticule-on Corneal Collagen Cross-Linking in Thin Keratoconic Corneas. Int. Ophthalmol. 40, 3403-3412. doi:10.1007/s10792020-01526-x

Caporossi, A., Baiocchi, S., Mazzotta, C., Traversi, C., and Caporossi, T. (2006). Parasurgical Therapy for Keratoconus by Riboflavin-Ultraviolet Type A Rays Induced Cross-Linking of Corneal Collagen. J. Cataract Refract. Surg. 32, 837-845. doi:10.1016/j.jcrs.2006.01.091

Cassagne, M., Pierné, K., Galiacy, S. D., Asfaux-Marfaing, M.-P., Fournié, P., and Malecaze, F. (2017). Customized Topography-Guided Corneal Collagen CrossLinking for Keratoconus. J. Refract Surg. 33, 290-297. doi:10.3928/1081597X20170201-02

Cha, S.-H., Lee, J.-S., Oum, B.-S., and Kim, C.-D. (2004). Corneal Epithelial Cellular Dysfunction from Benzalkonium Chloride (BAC) In Vitro. Clin. Exp. Ophthalmol. 32, 180-184. doi:10.1111/j.1442-9071.2004.00782.x

Chanbour, W., Mokdad, I., Mouhajer, A., and Jarade, E. (2019). Late-Onset Sterile Peripheral Ulcerative Keratitis Post-Corneal Collagen Crosslinking. Cornea 38, 338-343. doi:10.1097/ICO.0000000000001842

Choi, M., Kim, J., Kim, E. K., Seo, K. Y., and Kim, T.-I. (2017). Comparison of the Conventional Dresden Protocol and Accelerated Protocol with Higher Ultraviolet Intensity in Corneal Collagen Cross-Linking for Keratoconus. Cornea 36, 523-529. doi:10.1097/ICO.0000000000001165

Dawczynski, J., Franke, S., Blum, M., Kasper, M., Stein, G., and Strobel, J. (2002). Advanced Glycation End-Products in Corneas of Patients with Keratoconus. Graefe's Arch. Clin. Exp. Ophthalmol. 240, 296-301. doi:10.1007/s00417-0020445-3

Elbaz, U., Yeung, S. N., Ziai, S., Lichtinger, A. D., Zauberman, N. A., Goldich, Y., et al. (2014). Collagen Crosslinking After Radial Keratotomy. Cornea 33, 131-136. doi:10.1097/ICO.0000000000000044

Elling, M., Kersten-Gomez, I., and Dick, B. H. (2018). Photorefractive Intrastromal Corneal Crosslinking for Treatment of Myopic Refractive Error: Findings from 12-month Prospective Study Using an Epithelium-Off Protocol. J. Cataract Refract. Surg. 44, 487-495. doi:10.1016/j.jcrs.2018.01.022
Evangelista, C. B., and Hatch, K. M. (2018). Corneal Collagen Cross-Linking Complications. Semin. Ophthalmol. 33, 29-35. doi:10.1080/ 08820538.2017.1353809

Freeman, R. D. (1972). Oxygen Consumption by the Component Layers of the Cornea. J. Physiol. 225, 15-32. doi:10.1113/jphysiol.1972.sp009927

Gomes, J. A. P., Tan, D., Rapuano, C. J., Belin, M. W., Ambrósio, R., Guell, J. L., et al. (2015). Global Consensus on Keratoconus and Ectatic Diseases. Cornea 34, 359-369. doi:10.1097/ICO.0000000000000408

Gore, D. M., Leucci, M. T., Koay, S.-Y., Kopsachilis, N., Nicolae, M. N., Malandrakis, M. I., et al. (2021). Accelerated Pulsed High-Fluence Corneal Cross-Linking for Progressive Keratoconus. Am. J. Ophthalmol. 221, 9-16. doi:10.1016/j.ajo.2020.08.021

Gore, D. M., O'Brart, D., French, P., Dunsby, C., and Allan, B. D. (2015). Transepithelial Riboflavin Absorption in an Ex Vivo Rabbit Corneal Model. Invest. Ophthalmol. Vis. Sci. 56, 5006-5011. doi:10.1167/iovs.15-16903

Grentzelos, M. A., Liakopoulos, D. A., Siganos, C. S., Tsilimbaris, M. K., Pallikaris, I. G., and Kymionis, G. D. (2019). Long-term Comparison of Combined T-PTK and CXL (Cretan Protocol) Versus CXL with Mechanical Epithelial Debridement for Keratoconus. J. Refract Surg. 35, 650-655. doi:10.3928/ 1081597X-20190917-01

Hafezi, F., Dejica, P., and Majo, F. (2010). Modified Corneal Collagen Crosslinking Reduces Corneal Oedema and Diurnal Visual Fluctuations in Fuchs Dystrophy. Br. J. Ophthalmol. 94, 660-661. doi:10.1136/bjo.2009.162479

Hafezi, F., Kling, S., Gilardoni, F., Hafezi, N., Hillen, M., Abrishamchi, R., et al. (2021). Individualized Corneal Cross-Linking with Riboflavin and UV-A in Ultrathin Corneas: The Sub400 Protocol. Am. J. Ophthalmol. 224, 133-142. doi:10.1016/j.ajo.2020.12.011

Hafezi, F., Mrochen, M., Iseli, H. P., and Seiler, T. (2009). Collagen Crosslinking with Ultraviolet-A and Hypoosmolar Riboflavin Solution in Thin Corneas. J. Cataract Refract. Surg. 35, 621-624. doi:10.1016/j.jcrs.2008.10.060

Hafezi, F., and Randleman, J. B. (2014). PACK-CXL: Defining CXL for Infectious Keratitis. J. Refract Surg. 30, 438-439. doi:10.3928/1081597X-20140609-01

Hafezi, F., Richoz, O., Torres-Netto, E. A., Hillen, M., and Hafezi, N. L. (2021a). Corneal Cross-Linking at the Slit Lamp. J. Refract Surg. 37, 78-82. doi:10.3928/ 1081597X-20201123-02

Hafezi, F., Torres-Netto, E. A., and Hillen, M. J. P. (2021b). Re: Prajna et al.: CrossLinking-Assisted Infection Reduction: A Randomized Clinical Trial Evaluating the Effect of Adjuvant Cross-linking on Outcomes in Fungal Keratitis (Ophthalmology. 2020;127:159-166). Ophthalmology 128, e6-166. doi:10.1016/j.ophtha.2020.07.011

Hammer, A., Richoz, O., Mosquera, S. A., Tabibian, D., Hoogewoud, F., and Hafezi, F. (2014). Corneal Biomechanical Properties at Different Corneal CrossLinking (CXL) Irradiances. Invest. Ophthalmol. Vis. Sci. 55, 2881-2884. doi:10.1167/iovs.13-13748

Hammond, P., and Lobanoff, M. (2019). Update on Keratoconus Treatment. Mod. Optom. Available at: https://modernod.com/articles/2019-may/update-onkeratoconus-treatment ( (Accessed January 14, 2021).

Institute of Medicine US (1998). Dietary Reference Intakes for Thiamin, Riboflavin, Niacin, Vitamin B6, Folate, Vitamin B12, Pantothenic Acid, Biotin, and Choline. Washington, DC: National Academies Press (US).

Iseli, H. P., Popp, M., Seiler, T., Spoerl, E., and Mrochen, M. (2011). Laboratory Measurement of the Absorption Coefficient of Riboflavin for Ultraviolet Light (365 Nm). J. Refract Surg. 27, 195-201. doi:10.3928/1081597X-20100604-01

Jacob, S., Kumar, D. A., Agarwal, A., Basu, S., Sinha, P., and Agarwal, A. (2014). Contact Lens-Assisted Collagen Cross-Linking (CACXL): A New Technique for Cross-Linking Thin Corneas. J. Refract Surg. 30, 366-372. doi:10.3928/ 1081597X-20140523-01

Jacob, S., Patel, S. R., Agarwal, A., Ramalingam, A., Saijimol, A. I., and Raj, J. M. (2018). Corneal Allogenic Intrastromal Ring Segments (CAIRS) Combined with Corneal Cross-Linking for Keratoconus. J. Refract Surg. 34, 296-303. doi:10.3928/1081597X-20180223-01

Jarstad, J., M McDaniel, L., R Schaeffer, A., and A Taranissi, M. (2019). High-dose Dietary Riboflavin and Direct Sunlight Exposure in the Treatment of Keratoconus and Post-refractive Surgery Ectasia of the Cornea. Integr. Clin. Med. 3. doi:10.15761/ICM.1000160

Kandel, H., Nguyen, V., Ferdi, A. C., Gupta, A., Abbondanza, M., Sullivan, L., et al. (2021). Comparative Efficacy and Safety of Standard Versus Accelerated 
Corneal Crosslinking for Keratoconus. Cornea [Epub ahead of print]. doi:10.1097/ICO.0000000000002747

Kanellopoulos, A. J., and Asimellis, G. (2015). Combined Laser In Situ Keratomileusis and Prophylactic High-Fluence Corneal Collagen Crosslinking for High Myopia: Two-Year Safety and Efficacy. J. Cataract Refract. Surg. 41, 1426-1433. doi:10.1016/j.jcrs.2014.10.045

Kanellopoulos, A. J. (2019). Management of Progressive Keratoconus with Partial Topography-Guided PRK Combined with Refractive, Customized CXL - A Novel Technique: The Enhanced Athens Protocol. Opth 13, 581-588. doi:10.2147/OPTH.S188517

Kim, B. Z., Jordan, C. A., McGhee, C. N. J., and Patel, D. V. (2016). Natural History of Corneal Haze After Corneal Collagen Crosslinking in Keratoconus Using Scheimpflug Analysis. J. Cataract Refract. Surg. 42, 1053-1059. doi:10.1016/ j.jcrs.2016.04.019

Kim, C. Y., and Kim, M. K. (2019). Effect of Sequential Intrastromal Corneal Ring Segment Implantation and Corneal Collagen Crosslinking in Corneal Ectasia. Korean J. Ophthalmol. 33, 528-538. doi:10.3341/kjo.2019.0088

Kirgiz, A., Eliacik, M., and Yildirim, Y. (2019). Different Accelerated Corneal Collagen Cross-Linking Treatment Modalities in Progressive Keratoconus. Eye Vis. 6, 16. doi:10.1186/s40662-019-0141-6

Kling, S., and Hafezi, F. (2017). An Algorithm to Predict the Biomechanical Stiffening Effect in Corneal Cross-Linking. J. Refract Surg. 33, 128-136. doi:10.3928/1081597X-20161206-01

Kling, S., Hammer, A., Conti, A., and Hafezi, F. (2017). Corneal Cross-Linking with Riboflavin and UV-A in the Mouse Cornea In Vivo: Morphological, Biochemical, and Physiological Analysis. Trans. Vis. Sci. Tech. 6, 7. doi:10.1167/tvst.6.1.7

Kling, S., Richoz, O., Hammer, A., Tabibian, D., Jacob, S., Agarwal, A., et al. (2015). Increased Biomechanical Efficacy of Corneal Cross-Linking in Thin Corneas Due to Higher Oxygen Availability. J. Refract Surg. 31, 840-846. doi:10.3928/ 1081597X-20151111-08

Knyazer, B., Krakauer, Y., Tailakh, M. A., Achiron, A., Hecht, I., Lifshitz, T., et al. (2020). Accelerated Corneal Cross-Linking as an Adjunct Therapy in the Management of Presumed Bacterial Keratitis: A Cohort Study. J. Refract Surg. 36, 258-264. doi:10.3928/1081597X-20200226-02

Kobashi, H., Torii, H., Toda, I., Kondo, S., Itoi, M., and Tsubota, K. (2020). Clinical Outcomes of KeraVio Using Violet Light: Emitting Glasses and Riboflavin Drops for Corneal Ectasia: A Pilot Study. Br. J. Ophthalmol. Published Online First: 05 September 2020. doi:10.1136/bjophthalmol-2020-316974

Kobashi, H., and Tsubota, K. (2020). Accelerated Versus Standard Corneal CrossLinking for Progressive Keratoconus: A Meta-Analysis of Randomized Controlled Trials. Cornea 39, 172-180. doi:10.1097/ICO.0000000000002092

Kodavoor, S., Tiwari, N., and Ramamurthy, D. (2020). Profile of Infectious and Sterile Keratitis After Accelerated Corneal Collagen Cross-Linking for Keratoconus. Oman J. Ophthalmol. 13, 18-23. doi:10.4103/ojo.OJO_115_2018

Kohnen, T., Lwowski, C., Hemkeppler, E., de'Lorenzo, N., Petermann, K., Forster, R., et al. (2020). Comparison of Femto-LASIK with Combined Accelerated Cross-Linking to Femto-LASIK in High Myopic Eyes: A Prospective Randomized Trial. Am. J. Ophthalmol. 211, 42-55. doi:10.1016/ j.ajo.2019.10.024

Koller, T., Mrochen, M., and Seiler, T. (2009). Complication and Failure Rates after Corneal Crosslinking. J. Cataract Refract. Surg. 35, 1358-1362. doi:10.1016/ j.jcrs.2009.03.035

Konstantopoulos, A., Liu, Y.-C., Teo, E. P., Nyein, C. L., Yam, G. H., and Mehta, J. S. (2019). Corneal Stability of LASIK and SMILE When Combined with Collagen Cross-Linking. Trans. Vis. Sci. Tech.Vis. Sci. Technol. 8, 21. doi:10.1167/tvst.8.3.21

Kontadakis, G. A., Kankariya, V. P., Tsoulnaras, K., Pallikaris, A. I., Plaka, A., and Kymionis, G. D. (2016). Long-Term Comparison of Simultaneous TopographyGuided Photorefractive Keratectomy Followed by Corneal Cross-Linking Versus Corneal Cross-Linking Alone. Ophthalmology 123, 974-983. doi:10.1016/j.ophtha.2016.01.010

Kozobolis, V., Gkika, M., Sideroudi, H., Tsaragli, E., Lydataki, S., Naoumidi, I., et al. (2016). Effect of Riboflavin/UVA Collagen Cross-Linking on Central Cornea, Limbus and Intraocular Pressure. Experimental Study in Rabbit Eyes. Acta Med. (Hradec Kralove, Czech Repub.) 59, 91-96. doi:10.14712/ 18059694.2016 .96
Kymionis, G. D., Grentzelos, M. A., Karavitaki, A. E., Kounis, G. A., Kontadakis, G. A., Yoo, S., et al. (2010). Transepithelial Phototherapeutic Keratectomy Using a 213-nm Solid-State Laser System Followed by Corneal Collagen Cross-Linking with Riboflavin and UVA Irradiation. J. Ophthalmol. 2010, 1-3. doi:10.1155/ 2010/146543

Kymionis, G. D., Grentzelos, M. A., Kounis, G. A., Diakonis, V. F., Limnopoulou, A. N., and Panagopoulou, S. I. (2012a). Combined Transepithelial Phototherapeutic Keratectomy and Corneal Collagen Cross-Linking for Progressive Keratoconus. Ophthalmology 119, 1777-1784. doi:10.1016/ j.ophtha.2012.03.038

Kymionis, G. D., Kontadakis, G. A., Kounis, G. A., Portaliou, D. M., Karavitaki, A. E., Magarakis, M., et al. (2009). Simultaneous Topography-Guided PRK Followed by Corneal Collagen Cross-Linking for Keratoconus. J. Refract Surg. 25, S807-S811. doi:10.3928/1081597X-20090813-09

Kymionis, G. D., Portaliou, D. M., Diakonis, V. F., Kounis, G. A., Panagopoulou, S. I., and Grentzelos, M. A. (2012b). Corneal Collagen Cross-Linking with Riboflavin and Ultraviolet-A Irradiation in Patients with Thin Corneas. Am. J. Ophthalmol. 153, 24-28. doi:10.1016/j.ajo.2011.05.036

Kymionis, G. D., Tsiklis, N. S., Pallikaris, A. I., Kounis, G., Diakonis, V. F., Astyrakakis, N., et al. (2006). Long-term Follow-Up of Intacs for Post-LASIK Corneal Ectasia. Ophthalmology 113, 1909-1917. doi:10.1016/ j.ophtha.2006.05.043

Lamy, R., Chan, E., Zhang, H., Salgaonkar, V. A., Good, S. D., Porco, T. C., et al. (2013). Ultrasound-enhanced Penetration of Topical Riboflavin into the Corneal Stroma. Invest. Ophthalmol. Vis. Sci. 54, 5908-5912. doi:10.1167/ iovs.13-12133

Li, W., and Wang, B. (2017). Efficacy and Safety of Transepithelial Corneal Collagen Crosslinking Surgery versus Standard Corneal Collagen Crosslinking Surgery for Keratoconus: A Meta-Analysis of Randomized Controlled Trials. BMC Ophthalmol. 17, 262. doi:10.1186/s12886-017-0657-2

Li, Y., Chamberlain, W., Tan, O., Brass, R., Weiss, J. L., and Huang, D. (2016). Subclinical Keratoconus Detection by Pattern Analysis of Corneal and Epithelial Thickness Maps with Optical Coherence Tomography. J. Cataract Refract. Surg. 42, 284-295. doi:10.1016/j.jcrs.2015.09.021

Lin, J.-T., and Cheng, D.-C. (2017). Modeling the Efficacy Profiles of UV-Light Activated Corneal Collagen Crosslinking. PLoS One 12, e0175002. doi:10.1371/ journal.pone. 0175002

Matteoli, S., Virga, A., Paladini, I., Mencucci, R., and Corvi, A. (2016). Investigation into the Elastic Properties of Ex Vivo Porcine Corneas Subjected to Inflation Test After Cross-Linking Treatment. Jabfm 14, e163-170. doi:10.5301/ jabfm.5000262

Mazzotta, C., and Ramovecchi, V. (2014). Customized Epithelial Debridement for Thin Ectatic Corneas Undergoing Corneal Cross-Linking: Epithelial Island Cross-Linking Technique. Clin. Ophthalmol. 8, 1337-1343. doi:10.2147/ OPTH.S66372

Mazzotta, C., Bagaglia, S. A., Sgheri, A., Di Maggio, A., Fruschelli, M., Romani, A., et al. (2020a). Iontophoresis Corneal Cross-Linking with Enhanced Fluence and Pulsed UV-A Light: 3-Year Clinical Results. J. Refract Surg. 36, 286-292. doi:10.3928/1081597X-20200406-02

Mazzotta, C., and Caragiuli, S. (2014). Intraoperative Corneal Thickness Measurement by Optical Coherence Tomography in Keratoconic Patients Undergoing Corneal Collagen Cross-Linking. Am. J. Ophthalmol. 157, 1156-1162. doi:10.1016/j.ajo.2014.02.042

Mazzotta, C., Hafezi, F., Kymionis, G., Caragiuli, S., Jacob, S., Traversi, C., et al. (2015). In Vivo Confocal Microscopy After Corneal Collagen Crosslinking. Ocul. Surf. 13, 298-314. doi:10.1016/j.jtos.2015.04.007

Mazzotta, C., Raiskup, F., Hafezi, F., Torres-Netto, E. A., Armia Balamoun, A., Giannaccare, G., et al. (2021). Long Term Results of Accelerated $9 \mathrm{~mW}$ Corneal Crosslinking for Early Progressive Keratoconus: the Siena Eye-Cross Study 2. Eye Vis. 8, 16. doi:10.1186/s40662-021-00240-8

Mazzotta, C., Romani, A., and Burroni, A. (2018). Pachymetry-based Accelerated Crosslinking: The "M Nomogram" for Standardized Treatment of AllThickness Progressive Ectatic Corneas. Int. J. Keratoconus Ectatic Corneal Dis. 7, 137-144. doi:10.5005/jp-journals-10025-1171

Mazzotta, C., Sgheri, A., Bagaglia, S. A., Rechichi, M., and Di Maggio, A. (2020b). Customized Corneal Crosslinking for Treatment of Progressive Keratoconus: Clinical and OCT Outcomes Using a Transepithelial Approach with 
Supplemental Oxygen. J. Cataract Refract. Surg. 46, 1582-1587. doi:10.1097/ j.jcrs.0000000000000347

Mimouni, M., Sorkin, N., Sorkin, N., Trinh, T., Hatch, W., and Singal, N. (2021). KEI CXL Study Group, Central Versus Paracentral Cone Location and Outcomes of Accelerated Cross-Linking in Keratoconus Patients. Eye. doi:10.1038/s41433-021-01404-5

Moramarco, A., Iovieno, A., Sartori, A., and Fontana, L. (2015). Corneal Stromal Demarcation Line after Accelerated Crosslinking Using Continuous and Pulsed Light. J. Cataract Refract. Surg. 41, 2546-2551. doi:10.1016/j.jcrs.2015.04.033

Nabili, M., Geist, C., and Zderic, V. (2015). Thermal Safety of UltrasoundEnhanced Ocular Drug Delivery: A Modeling Study. Med. Phys. 42, 5604-5615. doi:10.1118/1.4929553

Naranjo, A., Arboleda, A., Martinez, J. D., Durkee, H., Aguilar, M. C., Relhan, N., et al. (2019). Rose Bengal Photodynamic Antimicrobial Therapy for Patients with Progressive Infectious Keratitis: A Pilot Clinical Study. Am. J. Ophthalmol. 208, 387-396. doi:10.1016/j.ajo.2019.08.027

Nath, S., Shen, C., Koziarz, A., Banfield, L., Nowrouzi-Kia, B., Fava, M. A., et al. (2020). Transepithelial Versus Epithelium-Off Corneal Collagen Cross-Linking for Corneal Ectasia. Ophthalmology. doi:10.1016/j.ophtha.2020.12.023

Ozbek-Uzman, S., Yalniz-Akkaya, Z., and Burcu, A. (2020). Corneal Collagen CrossLinking with Photoactivated Chromophore for Infectious Keratitis After Penetrating Keratoplasty. Cornea 39, 283-289. doi:10.1097/ICO.0000000000002210

Pandel, R., Poljšak, B., Godic, A., and Dahmane, R. (2013). Skin Photoaging and the Role of Antioxidants in its Prevention. ISRN Dermatol. 2013, 1-11. doi:10.1155/ 2013/9301642013

Peyman, A., Nouralishahi, A., Hafezi, F., Kling, S., and Peyman, M. (2016). Stromal Demarcation Line in Pulsed Versus Continuous Light Accelerated Corneal Cross-Linking for Keratoconus. J. Refract Surg. 32, 206-208. doi:10.3928/ 1081597X-20160204-03

Pitt, W. G., Husseini, G. A., and Staples, B. J. (2004). Ultrasonic Drug Delivery - A General Review. Expert Opin. Drug Deliv. 1, 37-56. doi:10.1517/17425247.1.1.37

Pollhammer, M., and Cursiefen, C. (2009). Bacterial Keratitis Early After Corneal Crosslinking with Riboflavin and Ultraviolet-A. J. Cataract Refract. Surg. 35, 588-589. doi:10.1016/j.jcrs.2008.09.029

Prajna, N. V., Radhakrishnan, N., Lalitha, P., Austin, A., Ray, K. J., Keenan, J. D., et al. (2020). Cross-Linking-Assisted Infection Reduction. Ophthalmology 127, 159-166. doi:10.1016/j.ophtha.2019.08.029

Raiskup, F., and Spoerl, E. (2011). Corneal Cross-Linking with Hypo-Osmolar Riboflavin Solution in Thin Keratoconic Corneas. Am. J. Ophthalmol. 152, 28-32. doi:10.1016/j.ajo.2011.01.016

Raiskup, F., and Spoerl, E. (2013). Corneal Crosslinking with Riboflavin and Ultraviolet A. I. Principles. Ocul. Surf. 11, 65-74. doi:10.1016/j.jtos.2013.01.002

Raiskup, F., Theuring, A., Pillunat, L. E., and Spoerl, E. (2015). Corneal Collagen Crosslinking with Riboflavin and Ultraviolet-A Light in Progressive Keratoconus: Ten-Year Results. J. Cataract Refract. Surg. 41, 41-46. doi:10.1016/j.jcrs.2014.09.033

Richoz, O., Hammer, A., Tabibian, D., Gatzioufas, Z., and Hafezi, F. (2013). The Biomechanical Effect of Corneal Collagen Cross-Linking (CXL) with Riboflavin and UV-A Is Oxygen Dependent. Trans. Vis. Sci. Tech. 2, 6. doi:10.1167/ tvst.2.7.6

Roberts, C. J., and Dupps, W. J. (2014). Biomechanics of Corneal Ectasia and Biomechanical Treatments. J. Cataract Refract. Surg. 40, 991-998. doi:10.1016/ j.jcrs.2014.04.013

Ross, A. H., Jones, M. N. A., Nguyen, D. Q., Jaycock, P. D., Armitage, W. J., Cook, S. D., et al. (2009). Long-term Topical Steroid Treatment After Penetrating Keratoplasty in Patients with Pseudophakic Bullous Keratopathy. Ophthalmology 116, 2369-2372. doi:10.1016/j.ophtha.2009.06.006

Sachdev, M. S., Gupta, D., Sachdev, G., and Sachdev, R. (2015). Tailored Stromal Expansion with a Refractive Lenticule for Crosslinking the Ultrathin Cornea. J. Cataract Refract. Surg. 41, 918-923. doi:10.1016/j.jcrs.2015.04.007

Salmon, B., Richoz, O., Tabibian, D., Kling, S., Wuarin, R., and Hafezi, F. (2017). CXL at the Slit Lamp: No Clinically Relevant Changes in Corneal Riboflavin Distribution During Upright UV Irradiation. J. Refract Surg. 33, 281. doi:10.3928/1081597X-20161219-03

Schindl, A., Rosado-Schlosser, B., and Trautinger, F. (2001). Die Reziprozitätsregel in der Photobiologie. Der Hautarzt 52, 779-785. doi:10.1007/s001050170065

Seifert, J., Hammer, C. M., Rheinlaender, J., Sel, S., Scholz, M., Paulsen, F., et al. (2014). Distribution of Young's Modulus in Porcine Corneas After Riboflavin/
UVA-Induced Collagen Cross-Linking as Measured by Atomic Force Microscopy. PLoS One 9, e88186. doi:10.1371/journal.pone.0088186

Seiler, T. G., Fischinger, I., Koller, T., Zapp, D., Frueh, B. E., and Seiler, T. (2016). Customized Corneal Cross-Linking: One-Year Results. Am. J. Ophthalmol. 166, 14-21. doi:10.1016/j.ajo.2016.02.029

Seiler, T. G., Komninou, M. A., Nambiar, M. H., Schuerch, K., Frueh, B. E., and Büchler, P. (2021). Oxygen Kinetics During Corneal Cross-Linking with and without Supplementary Oxygen. Am. J. Ophthalmol. 223, 368-376. doi:10.1016/ j.ajo.2020.11.001

Seyedian, M. A., Aliakbari, S., Miraftab, M., Hashemi, H., Asgari, S., and Khabazkhoob, M. (2015). Corneal Collagen Cross-Linking in the Treatment of Progressive Keratoconus: A Randomized Controlled Contralateral Eye Study. Middle East. Afr. J. Ophthalmol. 22, 340-345. doi:10.4103/0974-9233.159755

Sharma, A., Nottage, J. M., Mirchia, K., Sharma, R., Mohan, K., and Nirankari, V. S. (2012). Persistent Corneal Edema After Collagen Cross-Linking for Keratoconus. Am. J. Ophthalmol. 154, 922-926. doi:10.1016/j.ajo.2012.06.005

Sharma, N., Maharana, P., Singh, G., and Titiyal, J. S. (2010). Pseudomonas Keratitis After Collagen Crosslinking for Keratoconus: Case Report and Review of Literature. J. Cataract Refract. Surg. 36, 517-520. doi:10.1016/ j.jcrs.2009.08.041

Shi, C., Wang, M., Zhu, T., Zhang, Y., Ye, Y., Jiang, J., et al. (2020). Machine Learning Helps Improve Diagnostic Ability of Subclinical Keratoconus Using Scheimpflug and OCT Imaging Modalities. Eye Vis. 7, 48. doi:10.1186/s40662020-00213-3

Singal, N., Ong Tone, S., Stein, R., Bujak, M. C., Chan, C. C., Chew, H. F., et al. (2020). Comparison of Accelerated CXL Alone, Accelerated CXL-ICRS, and Accelerated CXL-TG-PRK in Progressive Keratoconus and Other Corneal Ectasias. J. Cataract Refract. Surg. 46, 276-286. doi:10.1097/ j.jcrs. 0000000000000049

Sitaula, S., Singh, S. K., and Gurung, A. (2019). Bilateral Viral Keratitis Following Corneal Collagen Crosslinking for Progressive Keratoconus. J. Ophthal Inflamm. Infect. 9, 16. doi:10.1186/s12348-019-0185-8

Soeters, N., Wisse, R. P. L., Godefrooij, D. A., Imhof, S. M., and Tahzib, N. G. (2015). Transepithelial Versus Epithelium-Off Corneal Cross-Linking for the Treatment of Progressive Keratoconus: A Randomized Controlled Trial. Am. J. Ophthalmol. 159, 821-828. doi:10.1016/j.ajo.2015.02.005

Sot, M., Gan, G., François, J., Chaussard, D., Da Costa, M., Luc, M. S., et al. (2021). Risk Factors for Keratoconus Progression after Treatment by Accelerated Cross-Linking (A-CXL): A Prospective 24-month Study. J. Français d'Ophtalmologie 44, 863-872. doi:10.1016/j.jfo.2020.08.040

Spoerl, E., Huhle, M., and Seiler, T. (1998). Induction of Cross-Links in Corneal Tissue. Exp. Eye Res. 66, 97-103. doi:10.1006/exer.1997.0410

Spoerl, E., Mrochen, M., Sliney, D., Trokel, S., and Seiler, T. (2007). Safety of UVARiboflavin Cross-Linking of the Cornea. Cornea 26, 385-389. doi:10.1097/ ICO.0b013e3180334f78

Stojanovic, A., Zhou, W., and Utheim, T. P. (2014). Corneal Collagen CrossLinking with and without Epithelial Removal: A Contralateral Study with 0.5\% Hypotonic Riboflavin Solution. Biomed. Res. Int. 2014, 619398. doi:10.1155/ 2014/619398

Taşçı, Y. Y., Taşlıpınar, G., Eyidoğan, D., Saraç, Ö., and Çağıl, N. (2020). Five-Year Long-Term Results of Standard Collagen Cross-Linking Therapy in Patients with Keratoconus. Turk. J. Ophthalmol. 50, 200-205. doi:10.4274/ tjo.galenos.2020.53810

Tawfeek, M., Ammar, K., Hosny, M., and Enany, H. (2020). "Photo-activated Chromophore for Keratitis (PACK-CXL) as Adjunctive Therapy for Infectious Keratitis: A Prospective Study," in 38th Congress of the European Society of Cataract and Refractive Surgeons. Available at: https://www.escrs.org/ amsterdam2020/programme/free-papers-details.asp?id=36752.

Ting, D. S. J., Henein, C., Said, D. G., and Dua, H. S. (2020). Re: Prajna et al.: CrossLinking-Assisted Infection Reduction (CLAIR): A randomized clinical trial evaluating the effect of adjuvant cross-linking on outcomes in fungal keratitis (Ophthalmology. 2020;127:159-166). Ophthalmology 127, e55-e56. doi:10.1016/j.ophtha.2020.02.032

Torres-Netto, E. A., Shetty, R., Knyazer, B., Chen, S., Hosny, M., Gilardoni, F., et al. (2020). "Corneal Cross-Linking for Treating Infectious Keratitis: Final Results of the Prospective Randomized Controlled Multicenter Trial," in 38th Congress of the ESCRS. Available at: https://www.escrs.org/amsterdam2020/programme/ posters-details.asp?id=35943. 
Tzamalis, A., Romano, V., Cheeseman, R., Vinciguerra, R., Batterbury, M., Willoughby, C., et al. (2019). Bandage Contact Lens and Topical Steroids Are Risk Factors for the Development of Microbial Keratitis After EpitheliumOff CXL. BMJ Open Ophth 4, e000231. doi:10.1136/bmjophth-2018-000231

Uddaraju, M., Mascarenhas, J., Das, M. R., Radhakrishnan, N., Keenan, J. D., Prajna, L., et al. (2015). Corneal Cross-Linking as an Adjuvant Therapy in the Management of Recalcitrant Deep Stromal Fungal Keratitis: A Randomized Trial. Am. J. Ophthalmol. 160, 131-134. doi:10.1016/ j.ajo.2015.03.024

Vajpayee, R. B., Shafi, S. N., Maharana, P. K., Sharma, N., and Jhanji, V. (2015). Evaluation of Corneal Collagen Cross-Linking as an Additional Therapy in Mycotic Keratitis. Clin. Exp. Ophthalmol. 43, 103-107. doi:10.1111/ceo.12399 van Dijk, K., Parker, J. S., Baydoun, L., Ilyas, A., Dapena, I., Groeneveld-van Beek, E. A., et al. (2018). Bowman Layer Transplantation: 5-year Results. Graefes Arch. Clin. Exp. Ophthalmol. 256, 1151-1158. doi:10.1007/s00417-018-3927-7

Vazirani, J., and Vaddavalli, P. K. (2013). Cross-linking for Microbial Keratitis. Indian J. Ophthalmol. 61, 441-444. doi:10.4103/0301-4738.116068

Wei, A., Wang, K., Wang, Y., Gong, L., Xu, J., and Shao, T. (2019). Evaluation of Corneal Cross-Linking as Adjuvant Therapy for the Management of Fungal Keratitis. Graefes Arch. Clin. Exp. Ophthalmol. 257, 1443-1452. doi:10.1007/ s00417-019-04314-1

Wen, D., Song, B., Li, Q., Tu, R., Huang, Y., Wang, Q., et al. (2018). Comparison of Epithelium-Off versus Transepithelial Corneal Collagen Cross-Linking for Keratoconus: A Systematic Review and Meta-Analysis. Cornea 37, 1018-1024. doi:10.1097/ICO.0000000000001632

Wernli, J., Schumacher, S., Spoerl, E., and Mrochen, M. (2013). The Efficacy of Corneal Cross-Linking Shows a Sudden Decrease with Very High Intensity UV Light and Short Treatment Time. Invest. Ophthalmol. Vis. Sci. 54, 1176-1180. doi:10.1167/iovs.12-11409

Wittig-Silva, C., Chan, E., Islam, F. M. A., Wu, T., Whiting, M., and Snibson, G. R. (2014). A Randomized, Controlled Trial of Corneal Collagen Cross-Linking in Progressive Keratoconus. Ophthalmology 121, 812-821. doi:10.1016/j.ophtha.2013.10.028

Wollensak, G., Aurich, H., Wirbelauer, C., and Pham, D.-T. (2009). Potential Use of Riboflavin/UVA Cross-Linking in Bullous Keratopathy. Ophthalmic Res. 41, 114-117. doi:10.1159/000187630

Wollensak, G., Aurich, H., Wirbelauer, C., and Sel, S. (2010). Significance of the Riboflavin Film in Corneal Collagen Crosslinking. J. Cataract Refract. Surg. 36, 114-120. doi:10.1016/j.jcrs.2009.07.044
Wollensak, G., Spoerl, E., Reber, F., and Seiler, T. (2004a). Keratocyte Cytotoxicity of riboflavin/UVA-Treatment In Vitro. Eye 18, 718-722. doi:10.1038/ sj.eye.6700751

Wollensak, G., Spoerl, E., and Seiler, T. (2003a). Riboflavin/ultraviolet-a-induced Collagen Crosslinking for the Treatment of Keratoconus. Am. J. Ophthalmol. 135, 620-627. doi:10.1016/s0002-9394(02)02220-1

Wollensak, G., Spoerl, E., Wilsch, M., and Seiler, T. (2004b). Keratocyte Apoptosis After Corneal Collagen Cross-Linking Using Riboflavin/UVA Treatment. Cornea 23, 43-49. doi:10.1097/00003226-200401000-00008

Wollensak, G., Spörl, E., and Herbst, H. (2019). Biomechanical Efficacy of Contact Lens-Assisted Collagen Cross-Linking in Porcine Eyes. Acta Ophthalmol. 97, e84-e90. doi:10.1111/aos.13828

Wollensak, G., Spörl, E., Reber, F., Pillunat, L., and Funk, R. (2003b). Corneal Endothelial Cytotoxicity of Riboflavin/UVA Treatment In Vitro. Ophthalmic Res. 35, 324-328. doi:10.1159/000074071

Zygoura, V., Birbal, R. S., van Dijk, K., Parker, J. S., Baydoun, L., Dapena, I., et al. (2018). Validity of Bowman Layer Transplantation for Keratoconus: Visual Performance at 5-7 Years. Acta Ophthalmol. 96, e901-e902. doi:10.1111/ aos. 13745

Conflict of Interest: Professor FH holds a patent on a UV light source (PCT/CH 2012/000090)

The remaining authors declare that the research was conducted in the absence of any commercial or financial relationships that could be construed as a potential conflict of interest.

The handling editor declared a shared affiliation with one of the authors CHLL at time of review.

Copyright (C) 2021 Wu, Lim, Lim, Wong, Hafezi, Manotosh and Lim. This is an open-access article distributed under the terms of the Creative Commons Attribution License (CC BY). The use, distribution or reproduction in other forums is permitted, provided the original author(s) and the copyright owner(s) are credited and that the original publication in this journal is cited, in accordance with accepted academic practice. No use, distribution or reproduction is permitted which does not comply with these terms. 